\title{
Relation-Guided Pre-Training for Open-Domain Question Answering
}

\author{
Ziniu Hu, Yizhou Sun, Kai-Wei Chang \\ University of California, Los Angeles \\ \{bull, yzsun, kwchang\}@cs.ucla.edu
}

\begin{abstract}
Answering complex open-domain questions requires understanding the latent relations between involving entities. However, we found that the existing QA datasets are extremely imbalanced in some types of relations, which hurts the generalization performance over questions with long-tail relations. To remedy this problem, in this paper, we propose a Relation-Guided Pre-Training (RGPT-QA) framework $^{1}$. We first generate a relational QA dataset covering a wide range of relations from both the Wikidata triplets and Wikipedia hyperlinks. We then pre-train a QA model to infer the latent relations from the question, and then conduct extractive QA to get the target answer entity. We demonstrate that by pretraining with propoed RGPT-QA techique, the popular open-domain QA model, Dense Passage Retriever (DPR), achieves 2.2\%, 2.4\%, and $6.3 \%$ absolute improvement in Exact Match accuracy on Natural Questions, TriviaQA, and WebQuestions. Particularly, we show that RGPT-QA improves significantly on questions with long-tail relations.
\end{abstract}

\section{Introduction}

Open domain question answering is a challenging task that answers factoid questions based on evidence in a large corpus (e.g., Wikipedia). Most open-domain QA systems follow retriever-reader pipeline (Chen et al., 2017), in which a retriever selects a subset of candidate entities and associated passages from the corpus that might contain the answer, then a reader extracts a text span from the passages as the answer. This process involves multiple entities that are relevant to answer the question. The QA system is required to extract these entities from the question and passages and identify the (latent) semantic relations between these entities in order to answer the question. For example, to

\footnotetext{
${ }^{1}$ Dataset and code are released at https://github. $\mathrm{com} / \mathrm{acbull/RGPT-QA}$.
}

answer the following question: "Where did Steph Curry play college basketball at?", the QA model is required to reason the implicit relation triplet $\langle$ Steph Curry, Educated At, Davidson College $\rangle$ to identify the correct answer.

To capture the relation knowledge required to answer questions, most QA systems rely on humanannotated supervised QA datasets. However, it is expensive and tedious to annotate a large set of QA pairs that cover enough relational facts for training a strong QA model. In addition, we showed that even for a large QA dataset like Natural Questions (Kwiatkowski et al., 2019), its training set only covers $16.4 \%$ of relations in WikiData (Vrandecic and Krötzsch, 2014) knowledge graph. Moreover, for those covered relations, the frequency distribution is imbalanced, i.e., $30 \%$ of relation types appear only once. Consequently, for the questions involving infrequent (a.k.a, long-tail) relations in the training set, the QA exact match accuracy is $22.4 \%$ lower than average. Such a biased relation distribution of existing QA datasets severely hurts the generalization of trained QA systems.

To improve the open-domain QA systems for questions with long-tail relations, in this paper, we propose RGPT-QA, a simple yet effective RelationGuided Pre-training framework for training QA models with augmented relationa facts from knowledge graph. The framework consists of two steps: 1) generate a relational QA dataset that covers a wide range of relations without human labeling; 2) pre-train a QA model to predict latent relations from questions and conduct extractive QA.

The key of our framework is to generate a relational QA dataset that align entities in Wikipedia passages with structured knowledge graph (e.g., WikiData). We call such a dataset Grounded Relational Wiki-Graph. In this graph, each edge indicates the relationship of two connected entities, and the edge is linked to a passage in Wikipedia describing this relationship. As WikiData knowledge 


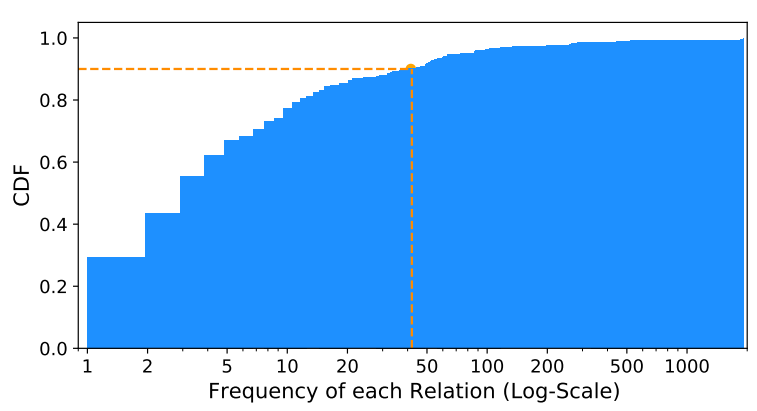

Figure 1: Cumulative distribution function (CDF) of relation frequency in Natural Question Training set.

graph also suffers from low coverage of long-tail entities and relations, we further convert hyperlinks in Wikipedia into knowledge triplets without specifying relation labels. Next, we link each relation triplet to a Wikipedia passage to help generate natural questions. We assume that if one passage in the Wiki-page of source entity contains the target entity, then the context in this passage describes the relationship between the two entities. With the constructed graph, we use a template to synthesize question and answer pairs and then pre-train the QA model to capture the relational facts for answering complex open-domain questions.

As a pre-training method, RGPT-QA can be incorporated with any open-domain QA system. In this paper, we utilize the recently developed Dense Passage Retriever (DPR) (Karpukhin et al., 2020) as the base QA system to evaluate the proposed pretraining effectiveness. Experimental results show that RGPT-QA enhances DPR's Exact Match accuracy by $2.2 \%, 2.4 \%$, and $6.3 \%$ on Natural Questions, TriviaQA and WebQuestions respectively. Compared with the existing QA pre-training methods (Lee et al., 2019; Guu et al., 2020a; Lewis et al., 2019), RGPT-QA explicitly captures a wide range of relational facts and thus achieves better performance. Moreover, for the questions containing long-tail relations in Natural Questions, the performance is improved by $10.9 \%$, showing that RGPT-QA alleviates the unbalanced relation distribution problem in the existing QA datasets.

The key contributions of this paper are:

- We propose RGPT-QA, a pre-training method to inject knowledge from relational facts in knowledge graph into QA models.

- RGPT-QA enhances the performance of a popular QA model, i.e., DPR, especially on the questions with long-tail relations.

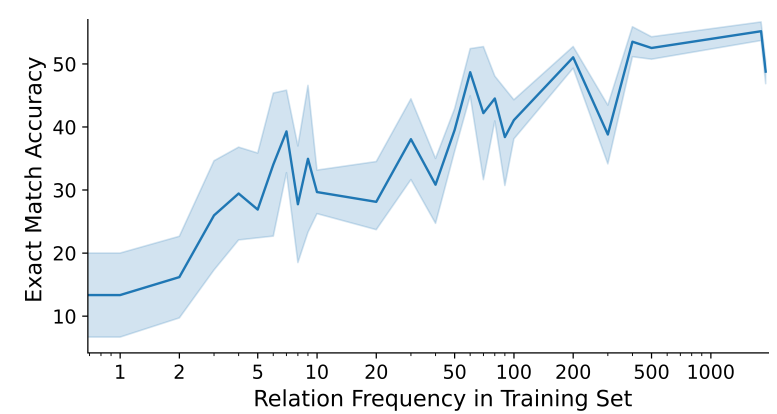

Figure 2: Exact Match accuracy of a trained DPR model in validation set with different relation frequency in training set.

\section{Preliminary and Empirical Analysis}

In this section, we firstly introduce the retrieverreader pipeline for open-domain QA, and then we analyze how the relation distribution in existing QA datsets influence generalization performance.

Open-Domain Question Answering. We focus on open-domain question answering that requires to extract answer from a large corpus (e.g. Wikipedia) $\mathbb{C}=\left\{p_{i}\right\}_{i=1}^{N}$ containing $N$ passages. Most open-domain QA systems follow a retrieverreader pipeline proposed by Chen et al. (2017). Given a factoid question $q$, the QA system first retrieves $K$ relevant passages $\left\{p_{j}\right\}_{j=1}^{K}$ from the corpus $\mathbb{C}$. Then a reading comprehension module extracts a text span $w_{\text {start }}, \ldots, w_{\text {end }}$ from one of these retrieved passages as the answer $a$ to the question. Some QA dataset annotated the passage where the answer $a$ is derived. We called this passage ground truth passage.

For the retriever, earlier systems utilize termbased retrieval methods, such as TF-IDF and BM25, which fails to capture the semantic relationship between question and passage beyond lexical matching. Recent studies (Lee et al., 2019; Karpukhin et al., 2020; Dhingra et al., 2020) use BERT-like pretrained language model to encode the question and passages independently into dense representations, and use maximum inner product search (MIPS) algorithms (Shrivastava and Li, 2014) to efficiently retrieve the most similar passage for each question. In this paper, we utilize Dense Passage Retriever (DPR) (Karpukhin et al., 2020) as the base QA model.

Relation Bias of Existing QA Datasets. We first explore how much relational knowledge between entities is required to answer the questions in the existing open-domain QA dataset. We con- 
duct an empirical study to analyze the relation distribution in Natural Questions, one of the largest open-domain QA datasets, and how it influences QA model's performance.

For each question in Natural Question training set, we first select the entity that the ground-truth passage is associated with. We then combine the entity with the answer as an entity pair, and check whether we can find a relation triplet in WikiData describing the relation between these two entities. Out of 58,880 training QA pairs, there are 23,499 pairs that could be aligned. The aligned QA pairs cover 329 relations, which accounts for $16.4 \%$ of the total 2,008 relations in WikiData. For most unaligned QA pairs, the answers are not entities and thus cannot be aligned to the graph.

In addition to the low relation coverage issue in Natural Question, we also find that the relation distribution is imbalanced. As showed in Figure 1, $90 \%$ of relations have frequency less than 41 , and $30 \%$ of relations appear only once. On the contrary, the most frequent relation "P161 (cast member)" appears 1,915 times out of 9,238 aligned QA pairs. A complete list of all these relations with aligned QA pairs is shown in Table 6-9 in Appendix.

We then study whether the imbalanced relation distribution influences the performance of QA models trained on these datasets. We use a DPR model trained on training set of Natural Questions and then calculate the Exact Match accuracy in validation set of each aligned QA pairs. We then analyze the correlation of the accuracy with the relation frequency in training set. As illustrated in Figure 2, the validation set accuracy is overall proportional to the relation frequency in training set. For those relations with frequency less than 5 , the average accuracy is only $20.3 \%$, much lower than the average accuracy $42.7 \%$ over all samples in validation set. This shows that the relation bias in existing QA datasets severely influences the generalization of QA models to questions with long-tail relations.

\section{Method}

In this section, we will discuss RGPT-QA framework in: 1) how to generate relational QA dataset for the pre-training purpose; and 2) how to construct a self-training task to empower QA model to capture relational facts.

\begin{tabular}{l|c}
\hline \# of linked Entity & $5,640,366$ \\
\# of relation labels & 2,008 \\
\# of labelled triplet & $14,463,728$ \\
\# of unlabeled triplet (hyperlink) & $66,796,110$ \\
\# of grounded descriptions per triplet & 1.25 \\
\hline
\end{tabular}

Table 1: Statistics of Grounded Relational Wiki-Graph.

\subsection{Construct QA Pre-Training Dataset}

To help QA model capture the knowledge from relation facts required to answer open-domain questions, we first focus on generating QA pre-training dataset, in which there exist relation connections between the source entity in questions to the target answer. Specifically, each QA pair datapoint $d=\left\langle\langle s, r, t\rangle, q, p^{+}\right\rangle$consists of three components: 1) relational triplet $\langle s, r, t\rangle$, in which $r$ denotes the relation between source entity $s$ and target entity $t$;2) question $q$ in natural language asking which entity has relation $r$ to source entity s, with target entity $t$ as the correct answer; 3 ) positive context passage $p^{+} \in \mathbb{C}[s]$, a passage from source entity's Wiki-page that contains the target answer $t$.

Grounded Relational Wiki-Graph. To generate QA pre-training dataset, leveraging the relation triplets in knowledge graph, e.g., WikiData, is a natural choice to define questions that require relation reasoning. We therefore construct Grounded Relational Wiki-Graph, in which each relation triplet $\langle s, r, t\rangle$ is linked to a set of description passages $\{$ desc. $(s, t)\}$ in the Wiki-page of entity $s$. These descriptions would be later utilized to generate questions $q$ and positive context passages $p^{+}$.

To construct such a graph, we use the 2021 Jan. English dump of Wikidata and Wikipedia. For each Wikipedia hyperlink $\langle s, ?, t\rangle$ (? denotes the relation is unlabeled), the passage containing anchored text to $t$ in the Wiki-page of $s$ naturally fits our requirement for desc. $(s, t)$. For each WikiData relation triplet $\langle s, r, t\rangle$, if the two entities are linked by a hyperlink in Wikipedia, we label the relation of the aligned hyperlink as $r$. For the other triplets $\langle s, r, t\rangle$ without alignment with hyperlinks, we extract all mentioning of target entity $t$ from the Wiki-page of $s$, and use the context passage as desc. $(s, t)$. The dataset statistics are shown in Table 1.

Relational QA Pair Generation In the following, we introduce the details to generate the relational QA pair from the constructed graph.

Recent unsupervised QA studies (Li et al., 2020; Pan et al., 2020) revealed that if the question $q$ and 


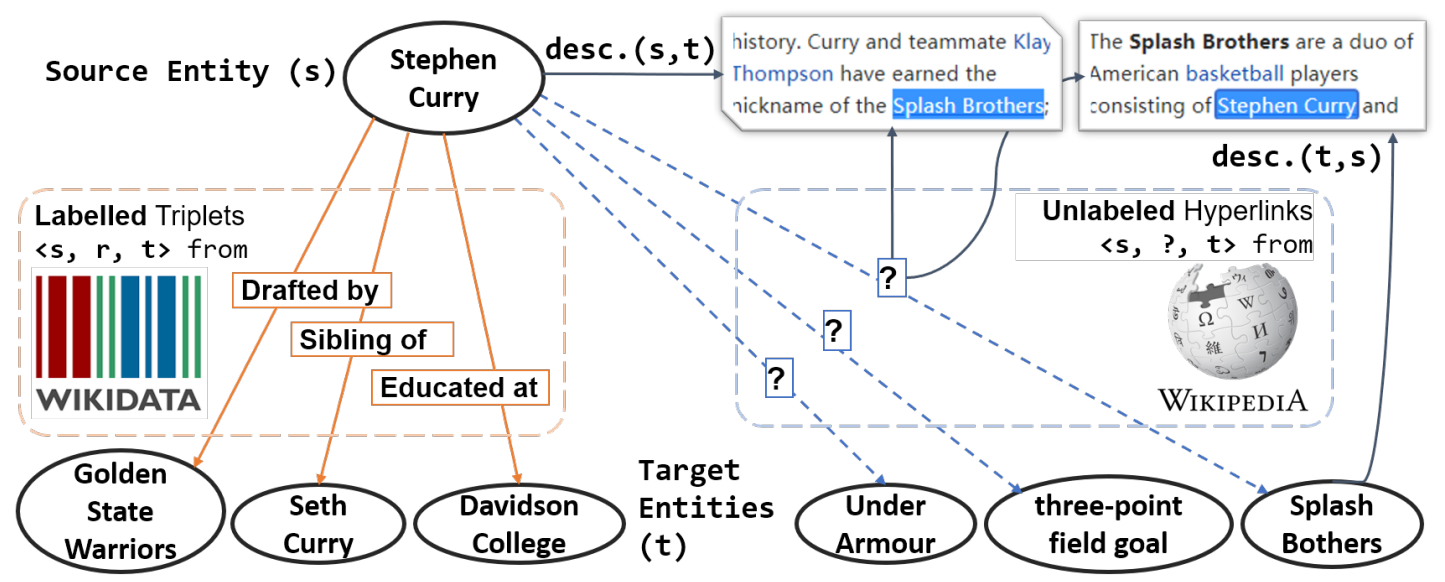

Relation Triplet: <Stephen Curry, ?, Splash Brothers>

Question: [<MASK $(r)>$ ] of [Stephen Curry] which [the $<\operatorname{MASK}(t)>$ which are a duo of American basketball players consisting of Stephen Curry and Klay Thompson].

True Passage: ...<MASK(s)> and teammate Klay Thompson have earned the nickname of the Splash Brothers...

Answer (Target Entity): Splash Brothers

Self-Distilled Relation Label: nickname $(p=0.18)$, member of team $(P=0.11)$, position on a team $(P=0.09) \ldots . .$.

Figure 3: Example of a generated relational QA pair from Grounded Relational Wiki-Graph.

context passage $p^{+}$share a large lexical overlap, then the QA model could utilize low-level lexical patterns as shortcuts to find the answer. These shortcuts hinder the model from learning to comprehend the passages and answer the questions, hurting model's generalizability. To avoid this lexical overlap issue, we aim to generate questions from a passage that is different from the context passage $p^{+}$.

We first select all the entity pairs $\langle s, t\rangle$ that have mutual links in the Grounded Relational WikiGraph, with desc. $(s, t)$ and $\operatorname{desc}(t, s)$ in part of Wikipage of $s$ and $t$ respectively, describing the relationship between the two entities. Without loss of generality, we denote $s$ as source entity and $t$ as the target answer. The passage desc. $(s, t)$ containing target answer $t$ can be used as the positive passage $p^{+}$.

Next, we generate a question that is lexically different from $p^{+}$using the following template:

$$
q(s, r, t)=[\operatorname{MASK}(r)] \text { of }[s] \text { which }[\text { desc. }(t, s)] ?
$$

in which MASK $(r)$ is a relation mask token. As desc. $(t, s)$ contains source entity $s$, it provides information to describe the relationship between $s$ and $t$, based on which the QA model should learn to infer the latent relation $r$, and retrieve positive passage $p^{+}=\operatorname{desc} .(s, t)$ and extract answer entity $t$. In addition, as desc. $(t, s)$ and desc. $(s, t)$ come from different Wiki-page, our question generation procedure can avoid the lexical overlap issue that often occur in prior Unsupervised QA methods.

Mask Target Answer. As description desc. $(t, s)$ is from target answer $t$ 's wiki-page, it often contains the name of entity $t$. We thus need to mask $t$ from the question. Otherwise, the pre-trained model can simply identify the answer to a question based on the local patterns.

As an example, in Figure 3, we show how to generate question for triplet 〈Stephen Curry, ?, Splash Brothers〉. We firstly retrieve two descriptive passages desc. $(s, t)$ and desc. $(t, s)$ in two entities' wiki pages. Using the template, we generate the question along with the ground-truth passage. We then mask out the target entity in question and source entity in true passage (will discuss later in retrieval pre-training) to avoid shortcut. A list of generated relational QA pairs are shown in Table 10 in Appendix.

\subsection{Relation-Guided QA Pre-Training}

With the generated relational QA dataset, we introduce how to pre-train both retriever and reader components in the QA model.

\subsubsection{Relation Prediction Pre-Training}

Our generated QA dataset contains the relation label $r$ between the source entity $s$ and the answer target $t$. Therefore, we design a self-training task to guide the model to predict the latent relation 
in question, which can benefit both retriever and reader. Specifically, we adopt a linear projection layer $\mathrm{L}_{R}(\cdot)$ over the $\mathrm{BERT}$ [CLS] token embedding to predict the relation over the WikiData relation set. The pre-training loss of relation prediction is:

$$
\mathcal{L}_{\text {rel }}=\frac{1}{B} \sum_{q}-\log P(r \mid q ; \theta),
$$

Self-Distillation for Unlabelled Relation The hyperlinks in wikipedia also provide valuable implicit information about the relations between entities. To leverage them, we use the trained relation predictor at each epoch with fixed parameter $\hat{\theta}$ as teacher model to assign soft label and then progressively train the relation predictor as student model based on the assigned labels in the next epoch. This approach is referred to as self-distillation in the literature (Xie et al., 2020; Chen et al., 2020). We minimize this self-distillation loss as:

$\mathcal{L}_{\text {distill }}=\frac{1}{B} \sum_{q} \sum_{\hat{r}}-\log P(\hat{r} \mid q ; \theta) \cdot \operatorname{sg}(P(\hat{r} \mid q ; \hat{\theta}))$,

where $\operatorname{sg}(\cdot)$ denotes the operation of stop gradient, which avoids back propagation to the teacher network with fixed parameter $\hat{\theta} . \hat{r}$ is enumerating all the relation labels.

As the relation predictor at early stages cannot give a reasonable prediction, we put a dynamic weight schedule to $\mathcal{L}_{\text {distill }}$ by a time-dependent weighting term $1-e^{- \text {epoch }}$, which ramps up from zero to one. Combing the weighted self-distillation loss $\mathcal{L}_{\text {distill }}$ with the supervised relation loss $\mathcal{L}_{\text {rel }}$, we get the final relation loss $\hat{\mathcal{L}}_{\text {rel }}$ to train the model capturing all relational facts covered in the Grounded Relational Wiki-Graph.

\subsubsection{Dense Retrieval Pre-Training}

The goal of dense retrieval pre-training is to get a question encoder $\operatorname{Enc}_{Q}$ and a passage encoder Enc $_{P}$ to map questions and all passages in the Wiki Corpus $\mathbb{C}$ into an embedding space, such that each question $q$ is close to its ground-truth positive context passage $p^{+}$in the embedding space. The objective is as follows:

$$
P_{\text {retr }}\left(p^{+} \mid q, \mathbb{C}\right)=\frac{\exp \left(\operatorname{sim}\left(q, p^{+}\right)\right)}{\sum_{p \in \mathbb{C}} \exp (\operatorname{sim}(q, p))},
$$

where $\operatorname{sim}(q, p)$ is the cosine similarity between the normalized embeddings of question and passage.
Two-Level Negative Passage Sampling. As we cannot enumerate all other passages in the denominator of $\mathrm{Eq}(1)$, we need to sample a set of negative passages for contrastive learning. Previous studies (Karpukhin et al., 2020) have revealed that it is essential that the sampled negative passages should be hard enough to train the retriever. As the question and passage embeddings are encoded independently, DPR can efficiently calculate the similarity of each question to all passages in the batch via dot product. Based on this property, as long as the passages within a batch are similar to each other, they serve the hard cases of negative passages to others. We thus propose a two-level negative passage sampling strategy to construct hard cases for training the retriever in the following.

We first sample at the level of entity. Given a set of randomly sampled $b$ entities, we adopt random walk from these seed entities over the Grounded Relational Wiki-Graph to get $B$ entities. As the connected entities have a relationship, their true passages are also semantically similar, and thus serve as good negative samples. We then conduct sampling at the level of passage. For each source entity $s_{i}$ with positive passage $p_{i}^{+} \in \mathbb{C}\left[s_{i}\right]$, we randomly pick $K$ other passages from the same Wiki-page to form a negative passage set $\left\{p_{i, j}^{-} \in\right.$ $\mathbb{C}\left[s_{i}\right]$, s.t. $\left.p_{i, j}^{-} \neq p_{i}^{+}\right\}_{j=1}^{K}$. These negative passages are similar to $p_{i}^{+}$, as they all describe the same entity $s_{i}$.

After we collect both the positive and $K$ negative passages for all the entities, we use the passage encoder $\operatorname{Enc}_{P}$ to get a passage embedding matrix $\mathbf{P}$ with dimension $((1+K) \cdot B \times d)$. We also use question encoder Enc $_{Q}$ to get question embedding matrix $\mathbf{Q}$ with dimension $(B \times d)$. We then get a similarity matrix $\mathbf{S}=\mathbf{Q} \mathbf{P}^{T}$ with dimension $(B \times(1+K) \cdot B)$, in which the diagonal entry corresponds to the similarity between question and its positive passage. We thus calculate the retrieval loss with in-batch negative samples via:

$$
\mathcal{L}_{\text {retr }}=\frac{1}{B}\left(\sum_{i \in[1, B]}(-\log \operatorname{softmax}(\mathbf{S}))_{[i, i]}\right) .
$$

Masking Source Entity. As the true passage $p_{i}^{+}=$desc. $(s, t)$ might contain the name of source entity $s$. We mask out all the tokens of $s$ from the extracted passages, so that the model is required to understand the passages for correct retrieval instead of exploiting a shortcut. 


\subsubsection{Reading Comprehension Pre-Training}

The goal of reading comprehension pre-training is to get a neural reader that re-ranks the top- $k$ retrieved passages and extracts an answer span from each passage as the answer. The probability of a passage contains the target answer $t$, and each token in the selected passage being the starting/ending positions of an $t$ are defined as:

$$
\begin{aligned}
& P_{\text {rank }}(t \in p)=\frac{\exp \left(\mathrm{L}_{\text {rank }}\left(\operatorname{BERT}_{\text {CLS }}(q, p)\right)\right)}{\sum_{\hat{p}} \exp \left(\mathrm{L}_{\text {rank }}\left(\operatorname{BERT}_{\mathrm{CLS}}(q, \hat{p})\right)\right)}, \\
& P_{\text {start }}(i \mid p, q)=\frac{\exp \left(\mathrm{L}_{\text {start }}\left(\operatorname{BERT}_{[i]}(q, p)\right)\right)}{\sum_{j} \exp \left(\mathrm{L}_{\text {start }}\left(\operatorname{BERT}_{[j]}(q, p)\right)\right)}, \\
& P_{\text {end }}(i \mid p, q)=\frac{\exp \left(\mathrm{L}_{\text {end }}\left(\operatorname{BERT}_{[i]}(q, p)\right)\right)}{\sum_{j} \exp \left(\mathrm{L}_{\text {end }}\left(\operatorname{BERT}_{[j]}(q, p)\right)\right)} .
\end{aligned}
$$

where $\mathrm{L}_{*}$ are linear project layers with different parameters. Note that the re-ranking module adopts cross-attention over questions and passages rather than the dot product of two independently encoded embedding used in retriever. For each QA pair $d=\left\langle\langle s, r, t\rangle, q, p^{+}\right\rangle$, we select $m$ other passages in wiki-page of entity $s$ as negative passages, and maximize $P_{\text {rank }}\left(t \in p^{+}\right)$. Then, we calculate $P_{\text {start }}\left(i \mid p^{+}, q\right)$ and $P_{\text {end }}\left(i \mid p^{+}, q\right)$ and maximize the probability for the ground-truth span of target answer $t$. Combing the passage re-ranking and span extraction objectives, we get readingcomprehension loss $\mathcal{L}_{\text {read }}$.

\section{Experiments}

In this section, we evaluate RGPT-QA on three open-domain QA datasets: Natural Questions (NQ), Trivia QA and Web Questions (WQ).

\subsection{Experiment Settings}

We follow the pre-processing procedure described in DPR (Karpukhin et al., 2020) for a fair comparison. We use the English Wikipedia from Dec. 20, 2018 and split each article into passages of 100 disjoint words as the corpus. For each question in all the three datasets, we use a passage from the processed Wikipedia which contains the answer as positive passages. We evaluate the QA system by Exact Match (EM) Accuracy on the correct answer.

Our RGPT-QA could be integrated with any open-domain QA system. In this paper, we incorporate it with the recently developed QA system, Dense Passage Retriever (DPR) (Karpukhin et al., 2020) to evaluate our pre-training framework. The DPR model uses the RoBERTa-base $(\mathrm{d}=768, \mathrm{l}=12)$ model as the base encoder. We first pre-train the retriever and reader in DPR using RGPT-QA. For retriever, we use the negative passage sampling strategy (c.f. Sec. 3.2.2), with initial entity size set to be 12 , batch size of 128 and the hard negative passage number of 2 . For reader, we randomly sample 64 source entities per batch to calculate the loss. For each entity, we sample 2 hard negative passages for re-ranking. We pre-train both the retriever and reader for 20 epochs using AdamW optimizer and a learning rate warm-up followed by linear decay. Pre-training is run on 8 Tesla V100 GPUs for two days. After the pre-training, we fine-tune the retriever and reader on each QA dataset following the same procedure and hyper-parameters described in DPR (Karpukhin et al., 2020).

QA Pre-Training Baselines. We compare RGPT-QA with three recently proposed pretraining methods for open-domain QA.

T5 (Raffel et al., 2020) adopts multiple generative tasks to pre-train a generative model. The fine-tuned QA models directly generate answers without needing an additional retrieval step.

ORQA (Lee et al., 2019) adopts a Inverse Cloze Task (ICT) to pre-train retriever, which forces each sentence's embedding close to context sentences.

REALM (Guu et al., 2020a) incorporates a retriever as a module into language model and trains the whole model over masked entity spans.

We directly report the results listed in their papers as they follow the same experiment settings.

We also add two knowledge-guided language models as baselines. Though not targeted at QA problem, these two methods are both designed to capture structured knowledge.

KnowBERT (Peters et al., 2019) adds entity embedding to each entity mention in text, and adopts the entity linking objective to pre-train the model.

KEPLER (Wang et al., 2019) uses Knowledge Embedding objective, i.e., TransE, to guide embedding encoded over entity description.

We initialize DPR base encoders by the released pre-trained models of these two work, and then finetune on each QA dataset with the same procedure.

We also add a Unsupervised Question Answering (Unsup.QA) (Lewis et al., 2019) as a baseline. For each entity as the answer, Unsup.QA selects a passage containing the entity as context passage and a cloze question. The cloze question is later rewritten by a machine translator to natural language. We use the generated QA dataset to pre-train both 


\begin{tabular}{|c|c|c|c|c|c|}
\hline & QA System Name & $\begin{array}{c}\text { Pre-Training } \\
\text { Task for QA }\end{array}$ & $\begin{array}{c}\text { NQ } \\
(58.9 \mathrm{k} / 3.6 \mathrm{k})\end{array}$ & $\begin{array}{c}\text { Trivia QA } \\
(60.4 \mathrm{k} / 11.3 \mathrm{k})\end{array}$ & $\begin{array}{c}\text { WQ } \\
(2.5 \mathrm{k} / 2 \mathrm{k})\end{array}$ \\
\hline 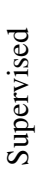 & $\begin{array}{l}\text { BM25+BERT (Lee et al., 2019) } \\
\text { HardEM (Min et al., 2019a) } \\
\text { GraphRetriever (Min et al., 2019b) } \\
\text { PathRetriever (Asai et al., 2020) } \\
\text { DPR (Karpukhin et al., 2020) }\end{array}$ & $\begin{array}{l}- \\
- \\
- \\
- \\
-\end{array}$ & $\begin{array}{l}26.5 \\
28.1 \\
34.5 \\
32.6 \\
41.5\end{array}$ & $\begin{array}{c}47.1 \\
50.9 \\
56.0 \\
- \\
56.8\end{array}$ & $\begin{array}{c}17.7 \\
- \\
36.4 \\
- \\
34.6\end{array}$ \\
\hline \multirow[t]{2}{*}{ 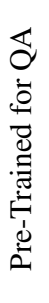 } & $\begin{array}{l}\text { T5 (large) (Raffel et al., 2020) } \\
\text { ORQA (Lee et al., 2019) } \\
\text { REALM }_{\text {Wiki }}(\text { Guu et al., 2020a) } \\
\text { REALM }_{\text {News }} \text { (Guu et al., 2020a) } \\
\text { DPR (KnowBERT (Peters et al., 2019)) } \\
\text { DPR (KEPLER (Wang et al., 2019)) } \\
\text { DPR (Unsup.QA (Lewis et al., 2019)) }\end{array}$ & $\begin{array}{c}\text { T5 (Multitask) } \\
\text { ICT } \\
\text { REALM } \\
\text { REALM } \\
\text { Entity Linking } \\
\text { TransE } \\
\text { Cloze Translation }\end{array}$ & $\begin{array}{l}29.8 \\
33.3 \\
39.2 \\
40.4 \\
39.1 \\
40.9 \\
41.9\end{array}$ & $\begin{array}{c}- \\
45.0 \\
- \\
- \\
56.4 \\
57.1 \\
57.3\end{array}$ & $\begin{array}{l}32.2 \\
36.4 \\
40.2 \\
40.7 \\
34.8 \\
35.2 \\
36.5\end{array}$ \\
\hline & Ours, DPR (RGPT-QA) & RGPT-QA & 43.7 & 59.2 & 40.9 \\
\hline
\end{tabular}

Table 2: End-to-end QA Exact Match Accuracy (\%) on test sets of three Open-Domain QA datasets, with the number of train/test examples shown in paretheses below. All the results except the last four rows are copied from the original papers. "-_ denotes no results are available. Models in the first block are initialized by BERT/RoBERTa and then directly fine-tuned on the supervised QA datasets. While models in the second block are initialized by RoBERTa and then tuned on some QA pre-training tasks first, and then fine-tuned on the supervised QA datasets.

the retriever and reader of the DPR framework.

\subsection{Experimental Results}

\begin{tabular}{l|ccc}
\hline Pre-Train Model & NQ & Trivia QA & WQ \\
\hline RoBERTa & $78.4 / 63.3$ & $79.4 / 72.6$ & $73.2 / 58.1$ \\
KnowBERT & $76.7 / 62.6$ & $78.9 / 72.2$ & $73.4 / 58.3$ \\
KEPLER & $77.9 / 62.8$ & $79.7 / 72.9$ & $74.5 / 58.6$ \\
Unsup.QA & $78.6 / 63.7$ & $79.9 / 73.0$ & $74.5 / 59.1$ \\
\hline RGPT-QA & $\mathbf{8 0 . 1} / \mathbf{6 4 . 8}$ & $\mathbf{8 1 . 2} / \mathbf{7 3 . 7}$ & $\mathbf{7 6 . 7 / 6 1 . 0}$ \\
\hline
\end{tabular}

Table 3: Retrieval (left) accuracy over Top-20 results and Reader (right) Exact Match over Golden-Passages on validation sets of three Open-Domain QA datasets.

\begin{tabular}{cccc|ccc}
\hline Mask & NPS & $\mathcal{L}_{\text {distill }}$ & $\mathcal{L}_{\text {rel }}$ & NQ & Trivia QA & WQ \\
\hline$\checkmark$ & $\checkmark$ & $\checkmark$ & $\checkmark$ & 44.3 & 59.8 & 41.4 \\
$\boldsymbol{X}$ & $\checkmark$ & $\checkmark$ & $\checkmark$ & 39.7 & 56.3 & 34.2 \\
$\checkmark$ & $\boldsymbol{X}$ & $\checkmark$ & $\checkmark$ & 43.5 & 58.1 & 39.8 \\
$\checkmark$ & $\checkmark$ & $\boldsymbol{X}$ & $\checkmark$ & 43.8 & 59.3 & 40.8 \\
$\checkmark$ & $\checkmark$ & $\boldsymbol{x}$ & $\boldsymbol{x}$ & 43.1 & 58.5 & 40.0 \\
\hline
\end{tabular}

Table 4: Ablation of RGPT-QA components on validation sets of three Open-Domain QA datasets. Mask: Mask target entity from question and source entity from passage; NPS: Two-level Negative Passage Sampling.

Table 2 summarizes the overall EM accuracy of the QA systems on the three datasets. The DPR framework pre-trained by RGPT-QA outperforms all other open-domain QA systems. Comparing with DPR without pre-training, RGPT-QA achieves $2.2 \%, 2.4 \%$ and $6.3 \%$ enhancement in EM accuracy on the three datasets.

\begin{tabular}{cc|ccc}
\hline B & K & NQ & Trivia QA & WQ \\
\hline 128 & 2 & 80.1 & 81.2 & 76.6 \\
128 & 1 & 79.7 & 80.8 & 76.1 \\
64 & 2 & 79.6 & 80.6 & 75.8 \\
64 & 1 & 79.2 & 80.1 & 75.3 \\
\hline
\end{tabular}

Table 5: Ablation of batch size and negative sampling for retrieval pre-training. B: Batch Size; K: Number of other passages as negative sample.

Comparing with other pre-training tasks for QA, RGPT-QA outperforms ORQA by $10.4 \%, 14.2 \%$ and $4.5 \%$ on the three datasets, and outperforms REALM $_{\text {News }}$ by $3.3 \%$ and $0.2 \%$ on NQ and WQ. This demonstrates that the model performance can be enhanced by leveraging relational QA dataset guided by Grounded Relational Wiki-Graph. We provide a detailed analysis in Sec. 4.3.

KnowBERT and KEPER encode structural knowledge into pre-trained language models. Both models focus on generating meaningful entity embedding, and are not designed to infer relations between entities for question answering. From the table, KEPLER trained via TransE performs slightly better than KnowBERT trained via entity linking, and RGPT-QA outperforms KEPLER by $2.8 \%, 2.1 \%, 5.7 \%$ on the three datasets.

Similar to RGPT-QA, Unsup.QA (Lewis et al., 2019) also generates QA data from Wikipedia. This baseline slightly improves DPR by $0.4 \%, 0.5 \%$, $1.9 \%$ on the three datasets, while our RGPT-QA outperforms it by $1.8 \%, 1.9 \%, 4.4 \%$. As discussed in Sec 3.1, one of the main reasons that 
our graph-based QA generation strategy performs better is that we adopt grounded description passages desc. $(t, s)$ and desc. $(s, t)$ from different documents as questions and contexts. This avoids the lexical overlap problem in Unsup.QA and help model to capture relational facts.

We also show the retrieval and reader performance separately on validation sets in Table 3 . Compared with DPR without pre-training, RGPTQA improves top-20 accuracy of Retriever by $1.7 \%$, $1.8 \%$, and $3.5 \%$, and improves EM accuracy of Reader by $1.5 \%, 1.1 \%$, and $2.9 \%$. Also, RGPTQA outperforms all the other pre-training baselines. This shows that RGPT-QA improves both the retrieval and reader steps of open-domain QA.

Ablation Studies. We then analyze the importance of each model component in RGPT-QA. One key strategy is to mask out the target answer from questions and mask out source entities from passages during retrieval training. This can avoid the model using the entity surface to find the correct passage and answer. Without using masking strategy, the average EM performance drops 5.1\%. This shows that it is essential to apply the mask strategy to avoid shortcut in QA pre-training. Next, we replace the hard negative passage sampling during retrieval pre-training with random batch sampling. The average EM performance drops 1.4\%, showing the importance of hard negative samples. Finally, we study the unsupervised relation loss $\mathcal{L}_{\text {distill }}$ and the supervised $\mathcal{L}_{\text {rel }}$. Removing them leads to $0.5 \%$ and $1.3 \%$ performance drop, which shows the benefit of training the model to explicitly infer the relation from questions.

Another key component is the negative passage sampling for dense retrieval pre-training. We study how the batch size and number of negative sample influence the performance of trained retrieval. As is shown in Table 5, increasing batch size and negative sample size can improve the performance of retriever. Even with a small batch size and negative sample, our pre-training framework could still achieves better performance against non-pretrain baseline, showing that our approach is not sensitive to these two hyperparameters.

Few-Shot QA Performance. We analyze the improvement of RGPT-QA when only a few labelled training samples are available. We fine-tune DPR initialized by RGPT-QA on subset of Natural Questions with different percentages. As is shown in

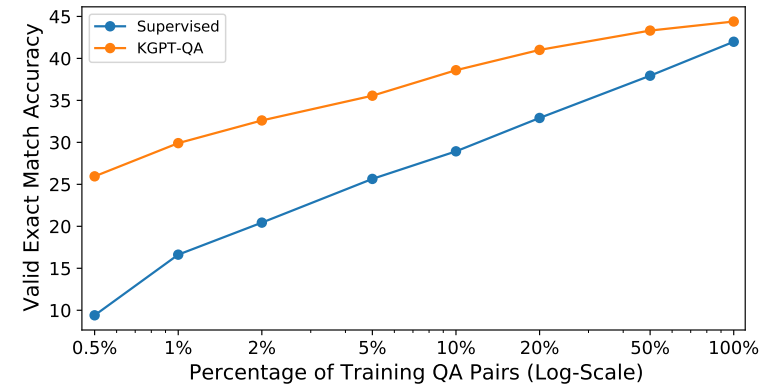

Figure 4: Few-shot QA experiment. Figure shows EM accuracy in validation set of DPR model with and without RGPT-QA pre-training, fine-tuned with different percentage of data on Natural Questions.

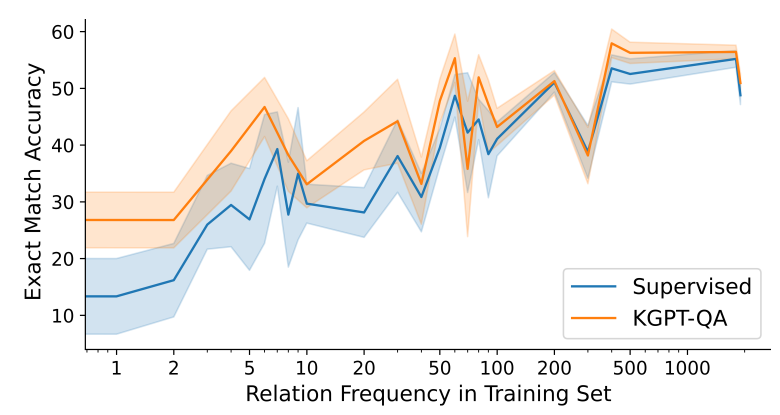

Figure 5: Long-tail relation experiment. EM accuracy of questions in validation set with different relation frequency in training set.

Figure 4, RGPT-QA consistently outperforms DPR without pre-training, and the improvement is more significant with small data. Specifically, when only $0.5 \%$ (594) labelled QA pairs are provided, the DPR pre-trained by RGPT-QA can still achieve $26.0 \%$ Val EM accuracy, significantly higher than 9.4\% achieved by the DPR without pre-training. The results show that RGPT-QA provides a good initialization for QA systems and reduce the requirement of large human-annotated QA dataset.

\subsection{Generalization for long-tail relations.}

As pointed out in Section 2, existing QA datasets suffer high relation bias, and thus a QA model trained on these datasets cannot generalize well to questions with long-tail relations. We thus analyze whether our RGPT-QA can remedy this issue. As is shown in Figure 5, the performance improvement of RGPT-QA against the supervised baseline is much more significant for the questions with infrequent relations. Specifically, for all relations appear less than 5 times in training set, the average EM accuracy of RGPT-QA is $33.3 \%$, significantly higher than $22.4 \%$ achieved by DPR without pre- 
training. This indicates that our relation QA generation method could indeed improve the performance on QA pairs with long-tail relations. Detailed prediction results are shown in Table 11 in Appendix.

\section{Related Works}

\section{Unsupervised QA via Question Generation} To train a QA system without human annotation of QA pairs, Unsupervised QA has been proposed by Lewis et al. (2019) to generate synthetic $\langle$ context, question, answer $\rangle$ data for training QA models. Lewis et al. (2019) synthesize the QA data by: 1) run NER or noun chunkers over randomly sampled English Wikipedia paragraphs to extract answers; 2) Treat the paragraphs surrounding the answer as context; 3) Treat the context as clozestyle question and feed into a unsupervised machine translator to generate natural questions. Some follow-up works also utilize template (Fabbri et al., 2020) and pre-trained language model (Puri et al., 2020) over masked cloze-style questions for more human-readable questions. These cloze-style unsupervised QA methods achieve promising performance than previous heuristic QA baselines but underperform supervised ones. The main limitation is that the question is generated with the masked context as input, resulting in severe overlap of lexicon and word surface with the context. Consequently, the QA model might utilize the lexical pattern as a shortcut to find the answer. To address the problem of context-question lexical overlap, Dhingra et al. (2018) assume each article has an introductory paragraph, and use this paragraph to generate answer. Li et al. (2020) retrieve the Wikipedia cited document as context, Pan et al. (2020) leverage structured tables to extract key information from context, with which to synthesize questions.

To tackle the challenges in previous studies, our framework propose to leverage the Wikipedia hyperlinks and Wikidata relations as the bridge to connect two entities with linked descriptions. With one description as question and the other as context, the question and context are semantically relevant and lexical different, which naturally solve the problem without involving any additional module.

Knowledge-Guided Pre-Training Recently, researchers investigated to inject structured knowledge into pre-trained language models. Zhang et al. (2019) and Peters et al. (2019) propose to add entity embedding to each entity mentions in text, and add entity linking objective to guide model capture structured knowledge. Wang et al. (2019) encode entity text description as entity embeddings and train them via TransE objective. Though these work show improvements over several natural language understanding tasks, they are not dedicated to open-domain question answering tasks.

There are also several pre-training studies for QA. For retrieval, Lee et al. (2019) propose an inverse cloze task, which treats a random sentence as query and the surrounding contexts as groundtruth evidence to train a QA retrieval model. Guu et al. (2020b) propose to explicitly add a retriever module in the language model to train the retriever via language modelling pre-training. For reader, Xiong et al. (2020) propose to a weakly supervised pre-training objective. They construct some fake sentences by replacing the entities in a sentence with the other entities of the same type, and train the model to discriminate original sentence from the fake ones. Verga et al. (2020) incorporate the knowledge graph triplets into language model, so the model could utilize the triplets to predict correct entity. Sun et al. (2021) extend this work by learning a virtual knowledge base by inferring the relation between two co-occurring entity pairs.

Compared with these works, our RGPT-QA mainly differs in: 1) We do not change the base QA model, so the pre-training framework could be applied to any QA systems. 2) We explicitly model the relations between entities, which proves to benefit QA pairs with less frequent relation patterns.

\section{Conclusion}

In this paper, we propose a simple yet effective pre-training framework RGPT-QA. We leverage both the Wikipedia hyperlinks and Wikidata relation triplets to construct Grounded Relational WikiGraph, based on which we generate relational QA dataset. We then pre-train a QA model to infer the latent relation from the question, and then conduct extractive QA to get the target answer entity. RGPT-QA improves the performance of the stateof-the-art QA frameworks, especially for questions with long-tail relations.

\section{Acknowledgement}

This work was partially supported by NSF III1705169, NSF 1937599, DARPA HR00112090027, Okawa Foundation Grant, and Amazon Research Awards. 


\section{References}

Akari Asai, Kazuma Hashimoto, Hannaneh Hajishirzi, Richard Socher, and Caiming Xiong. 2020. Learning to retrieve reasoning paths over wikipedia graph for question answering. In 8th International Conference on Learning Representations, ICLR 2020, Addis Ababa, Ethiopia, April 26-30, 2020. OpenReview.net.

Danqi Chen, Adam Fisch, Jason Weston, and Antoine Bordes. 2017. Reading wikipedia to answer opendomain questions. In Proceedings of the 55th Annual Meeting of the Association for Computational Linguistics, ACL 2017, Vancouver, Canada, July 30 August 4, Volume 1: Long Papers, pages 1870-1879. Association for Computational Linguistics.

Ting Chen, Simon Kornblith, Kevin Swersky, Mohammad Norouzi, and Geoffrey E. Hinton. 2020. Big self-supervised models are strong semi-supervised learners. In Advances in Neural Information Processing Systems 33: Annual Conference on Neural Information Processing Systems 2020, NeurIPS 2020, December 6-12, 2020, virtual.

Bhuwan Dhingra, Danish Pruthi, and Dheeraj Rajagopal. 2018. Simple and effective semi-supervised question answering. In Proceedings of the 2018 Conference of the North American Chapter of the Association for Computational Linguistics: Human Language Technologies, NAACL-HLT, New Orleans, Louisiana, USA, June 1-6, 2018, Volume 2 (Short Papers), pages 582-587. Association for Computational Linguistics.

Bhuwan Dhingra, Manzil Zaheer, Vidhisha Balachandran, Graham Neubig, Ruslan Salakhutdinov, and William W. Cohen. 2020. Differentiable reasoning over a virtual knowledge base. In 8th International Conference on Learning Representations, ICLR 2020, Addis Ababa, Ethiopia, April 26-30, 2020. OpenReview.net.

Alexander R. Fabbri, Patrick Ng, Zhiguo Wang, Ramesh Nallapati, and Bing Xiang. 2020. Templatebased question generation from retrieved sentences for improved unsupervised question answering. In Proceedings of the 58th Annual Meeting of the Association for Computational Linguistics, ACL 2020, Online, July 5-10, 2020, pages 4508-4513. Association for Computational Linguistics.

Kelvin Guu, Kenton Lee, Zora Tung, Panupong Pasupat, and Ming-Wei Chang. 2020a. REALM: retrieval-augmented language model pre-training. CoRR, abs/2002.08909.

Kelvin Guu, Kenton Lee, Zora Tung, Panupong Pasupat, and Ming-Wei Chang. 2020b. Retrieval augmented language model pre-training. In Proceedings of the 37th International Conference on Machine Learning, ICML 2020, 13-18 July 2020, Virtual Event, volume 119 of Proceedings of Machine Learning Research, pages 3929-3938. PMLR.
Vladimir Karpukhin, Barlas Oguz, Sewon Min, Ledell Wu, Sergey Edunov, Danqi Chen, and Wen-tau Yih. 2020. Dense passage retrieval for open-domain question answering. CoRR, abs/2004.04906.

Tom Kwiatkowski, Jennimaria Palomaki, Olivia Redfield, Michael Collins, Ankur P. Parikh, Chris Alberti, Danielle Epstein, Illia Polosukhin, Jacob Devlin, Kenton Lee, Kristina Toutanova, Llion Jones, Matthew Kelcey, Ming-Wei Chang, Andrew M. Dai, Jakob Uszkoreit, Quoc Le, and Slav Petrov. 2019. Natural questions: a benchmark for question answering research. Trans. Assoc. Comput. Linguistics, $7: 452-466$.

Kenton Lee, Ming-Wei Chang, and Kristina Toutanova. 2019. Latent retrieval for weakly supervised open domain question answering. In Proceedings of the 57th Conference of the Association for Computational Linguistics, ACL 2019, Florence, Italy, July 28-August 2, 2019, Volume 1: Long Papers, pages 6086-6096. Association for Computational Linguistics.

Patrick S. H. Lewis, Ludovic Denoyer, and Sebastian Riedel. 2019. Unsupervised question answering by cloze translation. In Proceedings of the 57th Conference of the Association for Computational Linguistics, ACL 2019, Florence, Italy, July 28-August 2, 2019, Volume 1: Long Papers, pages 4896-4910. Association for Computational Linguistics.

Zhongli Li, Wenhui Wang, Li Dong, Furu Wei, and Ke Xu. 2020. Harvesting and refining questionanswer pairs for unsupervised QA. In Proceedings of the 58th Annual Meeting of the Association for Computational Linguistics, ACL 2020, Online, July 5-10, 2020, pages 6719-6728. Association for Computational Linguistics.

Sewon Min, Danqi Chen, Hannaneh Hajishirzi, and Luke Zettlemoyer. 2019a. A discrete hard EM approach for weakly supervised question answering. In Proceedings of the 2019 Conference on Empirical Methods in Natural Language Processing and the 9th International Joint Conference on Natural Language Processing, EMNLP-IJCNLP 2019, Hong Kong, China, November 3-7, 2019, pages 28512864. Association for Computational Linguistics.

Sewon Min, Danqi Chen, Luke Zettlemoyer, and Hannaneh Hajishirzi. 2019b. Knowledge guided text retrieval and reading for open domain question answering. CoRR, abs/1911.03868.

Liangming Pan, Wenhu Chen, Wenhan Xiong, MinYen Kan, and William Yang Wang. 2020. Unsupervised multi-hop question answering by question generation. CoRR, abs/2010.12623.

Matthew E. Peters, Mark Neumann, Robert L. Logan IV, Roy Schwartz, Vidur Joshi, Sameer Singh, and Noah A. Smith. 2019. Knowledge enhanced contextual word representations. In Proceedings of the 2019 Conference on Empirical Methods in Natural Language Processing and the 9th International 
Joint Conference on Natural Language Processing, EMNLP-IJCNLP 2019, Hong Kong, China, November 3-7, 2019, pages 43-54. Association for Computational Linguistics.

Raul Puri, Ryan Spring, Mohammad Shoeybi, Mostofa Patwary, and Bryan Catanzaro. 2020. Training question answering models from synthetic data. In Proceedings of the 2020 Conference on Empirical Methods in Natural Language Processing, EMNLP 2020, Online, November 16-20, 2020, pages 5811-5826. Association for Computational Linguistics.

Colin Raffel, Noam Shazeer, Adam Roberts, Katherine Lee, Sharan Narang, Michael Matena, Yanqi Zhou, Wei Li, and Peter J. Liu. 2020. Exploring the limits of transfer learning with a unified text-to-text transformer. J. Mach. Learn. Res., 21:140:1-140:67.

Anshumali Shrivastava and Ping Li. 2014. Asymmetric LSH (ALSH) for sublinear time maximum inner product search (MIPS). In Advances in Neural Information Processing Systems 27: Annual Conference on Neural Information Processing Systems 2014, December 8-13 2014, Montreal, Quebec, Canada, pages 2321-2329.

Haitian Sun, Pat Verga, Bhuwan Dhingra, Ruslan Salakhutdinov, and William W. Cohen. 2021. Reasoning over virtual knowledge bases with open predicate relations. CoRR, abs/2102.07043.

Pat Verga, Haitian Sun, Livio Baldini Soares, and William W. Cohen. 2020. Facts as experts: Adaptable and interpretable neural memory over symbolic knowledge. CoRR, abs/2007.00849.

Denny Vrandecic and Markus Krötzsch. 2014. Wikidata: a free collaborative knowledgebase. Commun. ACM, 57(10):78-85.

Xiaozhi Wang, Tianyu Gao, Zhaocheng Zhu, Zhiyuan Liu, Juanzi Li, and Jian Tang. 2019. KEPLER: A unified model for knowledge embedding and pre-trained language representation. CoRR, abs/1911.06136.

Qizhe Xie, Minh-Thang Luong, Eduard H. Hovy, and Quoc V. Le. 2020. Self-training with noisy student improves imagenet classification. In 2020 IEEE/CVF Conference on Computer Vision and Pattern Recognition, CVPR 2020, Seattle, WA, USA, June 13-19, 2020, pages 10684-10695. IEEE.

Wenhan Xiong, Jingfei Du, William Yang Wang, and Veselin Stoyanov. 2020. Pretrained encyclopedia: Weakly supervised knowledge-pretrained language model. In 8th International Conference on Learning Representations, ICLR 2020, Addis Ababa, Ethiopia, April 26-30, 2020. OpenReview.net.

Zhengyan Zhang, Xu Han, Zhiyuan Liu, Xin Jiang, Maosong Sun, and Qun Liu. 2019. ERNIE: enhanced language representation with informative entities. In Proceedings of the 57th Conference of the Association for Computational Linguistics, ACL
2019, Florence, Italy, July 28- August 2, 2019, Volume 1: Long Papers, pages 1441-1451. Association for Computational Linguistics. 


\begin{tabular}{|c|c|c|c|}
\hline Relation & Frequency & Question & True Answer \\
\hline P161 (cast member) & 1915 & what was the geeks name in 16 candles & anthony michael hall \\
\hline P175 (performer) & 1844 & who sang the original blinded by the light & bruce springsteen \\
\hline P676 (lyrics by) & 519 & who sings the song $\mathrm{i}$ can see clearly now the rain is gone & johnny nash \\
\hline P86 (composer) & 442 & who made the beavis and butthead theme song & mike judge \\
\hline P725 (voice actor) & 334 & who plays the voice of tiana in princess and the frog & anika noni rose \\
\hline P1346 (winner) & 283 & who has won the 2017 womens singles wimbledon tennis tournament & garbiñe muguruza \\
\hline P50 (author) & 263 & where does the saying standing on the shoulders of giants come from & bernard of chartres \\
\hline P17 (country) & 257 & where did the black panther party take place & united states \\
\hline P527 (has part) & 198 & the unit of area in mks system is & metre \\
\hline P162 (producer) & 134 & who is in the video do $n$ 't worry be happy & bobby mcferrin \\
\hline P276 (location) & 117 & where will the summer olympics be held in 2020 & tokyo \\
\hline P840 (narrative location) & 103 & what state is a christmas story based in & indiana \\
\hline P915 (filming location) & 98 & where was the movie the english patient filmed & tunisia \\
\hline P710 (participant) & 88 & who died at the gunfight at okay corral & billy clanton \\
\hline P170 (creator) & 87 & who came up with britain 's got talent & simon cowell \\
\hline P1308 (officeholder) & 87 & who is the first lady of the usa & melania trump \\
\hline P361 (part of) & 74 & who sings if you want to destroy my sweater & weezer \\
\hline P39 ${ }_{r}$ (R: position held) & 64 & who is the attorney general for new jersey & gurbir grewal \\
\hline P138 (named after) & 64 & who proved that mar's orbit is elliptical not circular & nicolaus copernicus \\
\hline P112 (founded by) & 61 & who created a settlement house with the help of other social reformers & ellen gates starr \\
\hline $\mathrm{P} 161_{r}(\mathrm{R}:$ cast member $)$ & 60 & who is miss sue in the blind side & kathy bates \\
\hline $\mathrm{P} 31_{r}(\mathrm{R}:$ instance of $)$ & 57 & the world 's oldest epic tale told in poetry is called the epic of & epic of gilgamesh \\
\hline P58 (screenwriter) & 57 & who wrote the story for the shape of water & vanessa taylor \\
\hline P61 (discoverer or inventor) & 55 & who developed the analytical engine which had features of present day computers & charles babbage \\
\hline P26 (spouse) & 53 & who does young catherine marry in wuthering heights & hareton earnshaw \\
\hline P1923 (participating team) & 52 & who did the bengals play in the super bowl & san francisco $49 \mathrm{ers}$ \\
\hline $\mathrm{P} 166_{r}$ (R: award received) & 51 & which indian actor has won the most national awards & amitabh bachchan \\
\hline P674 (characters) & 50 & who said better to reign in hell than serve in heaven & satan \\
\hline P279 (subclass of) & 49 & when does dna replication occur during the eukaryotic cell cycle & mitosis \\
\hline $\mathrm{P} 361_{r}(\mathrm{R}:$ part of $)$ & 49 & where does the transmission of electrical impulses in the heart begin & sinoatrial node \\
\hline P131 (is located in) & 46 & where is saba university school of medicine located & saba \\
\hline $\mathrm{P} 279_{r}$ (R: subclass of $)$ & 45 & what are the names of the three pedals on a piano & soft pedal \\
\hline P54 (member of sports team) & 41 & what team does steph curry brother play for & dallas mavericks \\
\hline $\mathrm{P} 1344_{r}$ (R: participant in) & 38 & who won rupauls drag race all stars three & trixie mattel \\
\hline P495 (country of origin) & 37 & where was the movie snow white and the huntsman filmed & united kingdom \\
\hline P39 (position held) & 34 & who is the present speaker of lok sabha 2018 & sumitra mahajan \\
\hline P127 (owned by) & 33 & who owns the independent newspaper in the uk & alexander lebedev \\
\hline $\mathrm{P} 607_{r}(\mathrm{R}:$ conflict $)$ & 32 & in the civil war who had more soldiers & union army \\
\hline P31 (instance of) & 32 & what kind of bridge is the mackinac bridge & suspension bridge \\
\hline $\mathrm{P} 1441_{r}(\mathrm{R}:$ present in work) & 29 & what is the dads name in the adams family & gomez addams \\
\hline $\mathrm{P}^{175_{r}}(\mathrm{R}:$ performer $)$ & 28 & who does sean astin play in lord of the rings & samwise gamgee \\
\hline P36 (capital) & 28 & what is the capital of dadra and nagar haveli & silvassa \\
\hline P921 (main subject) & 24 & what disease did susannah have in brain on fire & anti-nmda receptor encephalitis \\
\hline P186 (material used) & 22 & what is the liquid in a magic 8 ball & alcohol \\
\hline $\mathrm{P} 179_{r}$ (R: part of the series) & 22 & what is the second book in the mortal instruments series & city of ashes \\
\hline $\mathrm{P} 793_{r}$ (R: significant event) & 21 & which territories did the us gain in the spanish-american war & puerto rico \\
\hline P115 (home venue) & 21 & where does portland 's nba basketball team the portland trailblazers play & moda center \\
\hline P371 (presenter) & 21 & who won beat bobby flay shrimp and grits & bobby flay \\
\hline P180 (depicts) & 19 & who $r$ the 4 presidents on $\mathrm{mt}$. rushmore & abraham lincoln \\
\hline $\mathrm{P} 800_{r}$ (R: notable work) & 19 & the explorer accurately mapped the coasts of europe and north africa & piri reis \\
\hline P136 (genre) & 17 & scott joplin is best known as a composer of what kind of music & ragtime \\
\hline P1431 (executive producer) & 16 & who hosted the daily show before trevor noah & jon stewart \\
\hline P47 (shares border with) & 16 & which indian states share a border with delhi & uttar pradesh \\
\hline P54 $4_{r}(\mathrm{R}:$ member of sports team) & 16 & who scored the first goal in dallas stars history & neal broten \\
\hline P144 (based on) & 16 & the tribute money depicts a scene from the & gospel of matthew \\
\hline P57 (director) & 15 & who is the director of welcome to new york & chakri toleti \\
\hline P488 (chairperson) & 15 & who is the leader of the democratic party now & tom perez \\
\hline P403 (watercourse outflow) & 15 & what sea does the nile river flow into & mediterranean sea \\
\hline P1889 (different from) & 14 & how to do alt codes on a mac & option key \\
\hline P1441 (present in work) & 14 & when does luke skywalker find out leia is his sister & return of the jedi \\
\hline P734 (family name) & 14 & who threw the first brick in the stonewall riots & johnson \\
\hline P1269 (facet of) & 14 & which supreme court case established the separate but equal doctrine & plessy v. ferguson \\
\hline P706 (takes place in) & 13 & what region of the world is greece in & southern europe \\
\hline P176 (manufacturer) & 13 & who built the gerald $\mathrm{r}$ ford aircraft carrier & newport news shipbuilding \\
\hline P84 (architect) & 12 & scottish architect who developed st martins in the field & james gibbs \\
\hline P150 (contains) & 12 & what is the name of capital of argentina & buenos aires \\
\hline P1532 (country for sport) & 12 & cristiano ronaldo what country does he play for & portugal \\
\hline P800 (notable work) & 12 & what was the first book that charles dickens published & the pickwick papers \\
\hline P641 (sport) & 11 & what is the number 1 sport in the usa & american football \\
\hline P1001 (applies to jurisdiction) & 11 & who won the schenck v. united states case & united states \\
\hline P206 (on lake) & 11 & where is ellis island located in new york & upper new york bay \\
\hline P178 (developer) & 11 & ms office 2000 was developed by which company & microsoft \\
\hline P166 (award received) & 11 & who won best actor in the academy awards this year & gary oldman \\
\hline $\mathrm{P} 102_{r}$ (R: party) & 11 & who was known as the father of indian national congress & mahatma gandhi \\
\hline P449 (original broadcaster) & 10 & what cbs channel is the late late show on & cbs \\
\hline P2438 (narrator) & 10 & whos the main character in the great gatsby & nick carraway \\
\hline P264 (record label) & 10 & who did the soundtrack for beverly hills cop & mca records \\
\hline $\mathrm{P} 674_{r}$ (R: characters) & 10 & where is the story of joseph in the bible found & book of genesis \\
\hline P1891 (signatory) & 10 & who has started reducing emissions from deforestation and forest degradation & brazil \\
\hline P138 ${ }_{r}$ (R: named after) & 10 & roman god of underworld also called orcus and pluto & pluto \\
\hline P69 (educated at) & 10 & where did jaren jackson senior play college basketball & georgetown university \\
\hline P1877 (after a work by) & 10 & the movie catch me if you can is based on who & frank abagnale \\
\hline
\end{tabular}

Table 6: Relation with grounded QA pairs of Natural Questions Training Set (Top 1-82 by frequency). 


\begin{tabular}{|c|c|c|c|}
\hline Relation & Frequency & Question & Answer \\
\hline P155 (follows) & 10 & what is the latest george rr martin book & a dance with dragons \\
\hline P1029 (crew member) & 10 & who was the first to step on moon & neil armstrong \\
\hline P3342 (significant person) & 10 & who was picked over kevin durant in the draft & greg oden \\
\hline P749 (parent organization) & 9 & what does chi mean in chi st lukes & catholic health initiatives \\
\hline P735 (given name) & 9 & who won in the war of alexander and porus & alexander \\
\hline $\mathrm{P} 463_{r}$ (R: member of) & 9 & countries in the warsaw pact during the cold war & soviet union \\
\hline P1376 (capital of) & 8 & cape town is the capital of what country & south africa \\
\hline P156 (followed by) & 8 & the things we do for love song artist & $10 \mathrm{cc}$ \\
\hline P451 (unmarried partner) & 8 & who does elena date in the vampire diaries & stefan salvatore \\
\hline P40 (child) & 8 & howard stark is the father of what superhero & iron man \\
\hline P159 (headquarters location) & 8 & where is the head office of rbi located & mumbai \\
\hline P287 (designed by) & 8 & who built the world first binary digit computer z1 & konrad zuse \\
\hline P551 (residence) & 8 & where did dorothy live in the wizard of oz & kansas \\
\hline P647 (drafted by) & 8 & who does dwyane wade play for in the nba & miami heat \\
\hline P30 (continent) & 8 & on what continents was the roman empire located at the height of its expansion & asia \\
\hline P634 (captain) & 7 & who is the captain of kolkata knight riders & dinesh karthik \\
\hline P828 (has cause) & 7 & what is the most common manifestation of portal hypertension - induced splenomegaly & cirrhosis \\
\hline P123 (publisher) & 7 & who made all the call of duty games & activision \\
\hline P2408 (set in period) & 7 & when did hunchback of notre dame take place & 1482 \\
\hline $\mathrm{P} 27_{r}$ (R: country of citizenship) & 7 & who was the last ruler of the tang dynasty & emperor ai of tang \\
\hline $\mathrm{P} 135_{r}$ (R: movement) & 7 & who wanted the catholic church to reform and address & martin luther \\
\hline $\mathrm{P} 101_{r}$ (R: field of work) & 7 & who invented the steam engine in the 1800 s & james watt \\
\hline P941 (inspired by) & 6 & who does squealer in animal farm represent in the russian revolution & vyacheslav molotov \\
\hline P136 (R: genre) & 6 & who are the founding fathers of hip hop & grandmaster flash \\
\hline P466 $r$ (R: occupant) & 6 & where did the patriots play before gillette stadium & foxboro stadium \\
\hline $\mathrm{P} 119_{r}(\mathrm{R}:$ place of burial) & 6 & who is buried in the great mausoleum at forest lawn glendale & michael jackson \\
\hline P88 (commissioned by) & 5 & who built the castle in just one day & toyotomi hideyoshi \\
\hline P110 (illustrator) & 5 & scary stories to tell in the dark artist & stephen gammell \\
\hline P1366 (replaced by) & 5 & the old greek city-state of byzantium was rebuilt and became known as & constantinople \\
\hline P169 (chief executive officer) & 5 & who become the ceo indian it company wipro in 2016 & abidali neemuchwala \\
\hline P3279 (statistical leader) & 5 & who is the captain of argentina national team fifa world cup 2018 & lionel messi \\
\hline P2388 (leader's office) & 5 & who does the us department of justice report to & united states attorney general \\
\hline $\mathrm{P} 53_{r}$ (R: family) & 5 & who began the first dynasty of egyptian rulers & narmer \\
\hline $\mathrm{P} 2522_{r}$ (R: victory) & 5 & who won season 2 of food network star & guy fieri \\
\hline P823 (speaker) & 5 & who wrote we shall fight on the beaches & winston churchill \\
\hline P748 (appointed by) & 5 & who can appoint comptroller and auditor general of india & president of india \\
\hline P1363 (points/goal scored by) & 5 & who scored the winning goal for england in the 1966 world cup final & geoff hurst \\
\hline P22 (father) & 5 & who was the king after david in the bible & solomon \\
\hline P1027 (conferred by) & 5 & who presents national film award traditionally in india & directorate of film festivals \\
\hline P750 (distributed by) & 5 & who own the rights to the black panther movie & walt disney studios motion pictures \\
\hline P825 (dedicated to) & 5 & who was the song candle in the wind written about & marilyn monroe \\
\hline P974 (tributary) & 5 & a tributary flowing into the mississippi from the east is the & ohio river \\
\hline P8031 (perpetrator) & 4 & who was the guy who shoot in las vegas & stephen paddock \\
\hline P885 (river source) & 4 & what is the starting point of the mississippi river & lake itasca \\
\hline P631 (structural engineer) & 4 & who designed the first tunnel under the river thames & marc isambard brunel \\
\hline $\mathrm{P} 17_{r}$ (R: country) & 4 & what are the countries of the united arab emirates & sharjah \\
\hline P98 (editor) & 4 & who was an abolitionist who published and autobiography and anti-slavery newspaper & frederick douglass \\
\hline P737 (influenced by) & 4 & qbasic is the extension of which programming language & quickbasic \\
\hline $\mathrm{P}^{206} 6_{r}$ (R: on lake) & 4 & where does the river mekong start and end & mekong delta \\
\hline P2789 (connects with) & 4 & a ship traveling through the panama canal could be crossing from the & atlantic ocean \\
\hline P740 (location of formation) & 4 & where did the beatles started their career as a band & liverpool \\
\hline P4743 (animal breed) & 4 & what kind of dog is bo and sunny & portuguese water dog \\
\hline P466 (occupant) & 4 & who used to play in the alamo dome & utsa roadrunners \\
\hline $\mathrm{P} 2868_{r}(\mathrm{R}:$ subject has role) & 4 & who is the commander in chief of military & president of the united states \\
\hline P5053 (fastest lap) & 4 & who won the 2018 chinese formula 1 grand prix & daniel ricciardo \\
\hline P106 $r$ (R: occupation) & 4 & who is the griot that sings the epic & balla fasséké \\
\hline $\mathrm{P} 0_{r}$ (R: author) & 4 & what is the title of langston hughes 's first book of poetry & the weary blues \\
\hline P118 (league) & 4 & what conference is ohio state in for football & big ten conference \\
\hline $\mathrm{P} 2416_{r}$ (R: sport discipline) & 4 & who has the world record for the long jump & galina chistyakova \\
\hline P1552 (has quality) & 4 & which metal does the word' ferrous 'refer to answer in words not symbols & iron \\
\hline P8111 (unit) & 4 & unit of measure for area of a triangle & square metre \\
\hline P179 (part of the series) & 4 & which games are in crash bandicoot $n$ sane trilogy & crash bandicoot \\
\hline $\mathrm{P} 131_{r}(\mathrm{R}:$ is located in) & 4 & what is the name of capital of andhra pradesh & amaravati \\
\hline P7047 (enemy of) & 4 & who sent doomsday to the end of time & superman \\
\hline $\mathrm{P} 725_{r}$ (R: voice actor) & 3 & who does the voice of the cat in the hat & martin short \\
\hline $\mathrm{P} 61_{r}$ (R: discoverer or inventor) & 3 & who discover the simple microscope first time and when & zacharias janssen \\
\hline P6 (head of government) & 3 & who was the founder of the mauryan empire & chandragupta maurya \\
\hline $\mathrm{P} 264_{r}(\mathrm{R}:$ record label) & 3 & this artist was signed in 1952 by atlantic and brought a string of hits & ray charles \\
\hline P462 (color) & 3 & what color was the white house when it was built & white \\
\hline P533 (target) & 3 & who was killed in the ides of march & julius caesar \\
\hline P972 (catalog) & 3 & who is on the top ten most wanted & alexis flores \\
\hline P1344 (participant in) & 3 & india 's first olympic medal win as a free nation & 1948 summer olympics \\
\hline P106 (occupation) & 3 & what did pete best play in the beatles & drummer \\
\hline $\mathrm{P} 1366_{r}$ (R: replaced by) & 3 & what is the old name for south africa & union of south africa \\
\hline $\mathrm{P} 171_{r}$ (R: parent taxon) & 3 & what type of organism is made up of prokaryotic cells & archaea \\
\hline P1411 (nominated for) & 3 & who won best director at the academy awards & guillermo del toro \\
\hline P8345 (media franchise) & 3 & what is the first star wars movie in the series & star wars \\
\hline P1433 (published in) & 3 & the story of seven ages by william shakespeare & as you like it \\
\hline $\mathrm{P} 20_{r}$ (R: place of death) & 3 & who was the explorer that reached the cape of good hope at the southern tip of africa & bartolomeu dias \\
\hline P87 (librettist) & 3 & who wrote the libretto for dido and aeneas & nahum tate \\
\hline P3764 (pole position) & 3 & who won the abu dhabi grand prix 2017 & valtteri bottas \\
\hline P559 (terminus) & 3 & what is the southern end of the appalachian trail & springer mountain \\
\hline
\end{tabular}

Table 7: Relation with grounded QA pairs of Natural Questions Training Set (Top 83-164 by frequency). 


\begin{tabular}{|c|c|c|c|}
\hline Relation & Frequency & Question & Answer \\
\hline P366 (use) & 3 & what did the chinese use oracle bones for & pyromancy \\
\hline $\mathrm{P} 06_{r}$ (R: takes place in) & 3 & what seven countries make up the subcontinent of south asia & sri lanka \\
\hline P610 (highest point) & 3 & what is the highest point in the pyrenees mountains in france & aneto \\
\hline P461 (opposite of) & 3 & the results of dehydration reactions can be reversed by & hydration reaction \\
\hline P467 (legislated by) & 3 & which group is responsible for adopting the declaration of independence & second continental congress \\
\hline P272 (production company) & 3 & what is the tv show riverdale based off of & archie comics \\
\hline $\mathrm{P}^{140_{r}}$ (R: religion) & 3 & who is the leader of the baptist denomination & thomas helwys \\
\hline P1419 (shape) & 3 & what is the shape of the earth 's orbit around the sun & ellipse \\
\hline P942 $(\mathrm{R}$ : theme music) & 3 & clubs who sing you 'll never walk alone & liverpool f.c. \\
\hline P376 (planet) & 3 & this planet is home to the great red spot & jupiter \\
\hline P5202 (adapted by) & 3 & who wrote the lyrics for the song my way & paul anka \\
\hline P171 (parent taxon) & 3 & trees of the betel nut genus of palms & areca \\
\hline P509 (cause of death) & 3 & what was the cause of the tollund man 's death & hanging \\
\hline P527 $r$ (R: has part) & 3 & the bronchi are considered to be part of the & respiratory system \\
\hline P2849 (produced by) & 3 & where does red blood cell formation occur in adults & bone marrow \\
\hline P460 (said to be the same as) & 3 & the word zion is an ancient biblical term that referred to what city & jerusalem \\
\hline $\mathrm{P} 1346_{r}$ (R: winner) & 3 & when did the philadelphia eagles last win the super bowl & super bowl lii \\
\hline P2341 (indigenous to) & 3 & dogri language is spoken in which state of india & himachal pradesh \\
\hline P355 (subsidiary) & 3 & the main agency under the department of homeland security that is responsible for border security is & u.s. customs and border protection \\
\hline P457 (foundational text) & 3 & where does one look to find the powers of a corporation & articles of incorporation \\
\hline P108 $(\mathrm{R}:$ employer) & 3 & who is the current ceo of mcdonald 's corporation & steve easterbrook \\
\hline $\mathrm{P} 1923_{r}$ (R: participating team) & 3 & last time houston astros have been to the world series & 2017 world series \\
\hline $\mathrm{P} 8345_{r}$ (R: media franchise) & 2 & what star wars movie came out before the last jedi & the empire strikes back \\
\hline $\mathrm{P} 112_{r}(\mathrm{R}:$ founded by $)$ & 2 & real name of raj chandra in rani rashmoni & babughat \\
\hline P113 ${ }_{r}$ (R: airline hub) & 2 & what airline has its hub in charlotte nc & american airlines \\
\hline $\mathrm{P} 156_{r}$ (R: followed by) & 2 & what is the origin of the coptic language & egyptian language \\
\hline P137 (operator) & 2 & who owns the white house in washington dc & national park service \\
\hline $\mathrm{P} 1552_{r}$ (R: has quality) & 2 & what physical quantity is a measure of the amount of inertia and object has & mass \\
\hline P2175 (disease treated) & 2 & topiramate ( topamax trokendi ) is used to treat which of the following diseases & epilepsy \\
\hline P25 (mother) & 2 & who is carries mother on days of our lives & anna dimera \\
\hline $\mathrm{P} 170_{r}$ (R: creator) & 2 & when was beverly cleary 's first book published & henry huggins \\
\hline P641 $r$ (R: sport) & 2 & where do the rocks from curling come from & ailsa craig \\
\hline $\mathrm{P} 451_{r}$ (R: unmarried partner) & 2 & who does raven end up with in the comics & beast boy \\
\hline P4584 (first appearance) & 2 & what was the first game waluigi was in & mario tennis \\
\hline P2670 (has parts of the class) & 2 & what do you rest a golf ball on & tee \\
\hline P1040 (film editor) & 2 & who is the director of the film avatar & james cameron \\
\hline $\mathrm{P} 1056_{r}$ (R: material produced) & 2 & who introduced the first micro processor in 1971 & intel \\
\hline $\mathrm{P} 1192_{r}$ (R: connecting service) & 2 & where does the eurostar leave from in paris & gare du nord \\
\hline P1830 (owner of) & 2 & where do the carolina panthers play home games & bank of america stadium \\
\hline P241 $r$ (R: military branch) & 2 & who served as the general of confederate forces during the civil war & robert e. lee \\
\hline P111 (measure of) & 2 & joule is unit of . in mks system & energy \\
\hline P19 $r$ (R: place of birth) & 2 & who was the last person to live in versaille & louis $\mathrm{xvi}$ \\
\hline P291 (place of publication) & 2 & where was the institutes of the christian religion published & basel \\
\hline P1056 (material produced) & 2 & by product of saponification of fats and oils & soap \\
\hline P140 (religion) & 2 & of which religion is the avesta a sacred book & zoroastrianism \\
\hline P137r (R: operator) & 2 & where do the fisher cats play in nh & northeast delta dental stadium \\
\hline $\mathrm{P} 162_{r}$ (R: producer) & 2 & producer and director of silence of the lambs & edward saxon \\
\hline P1582 (fruit of (taxon)) & 2 & a plant that produces a type of bean & fabaceae \\
\hline P286 (head coach) & 2 & 2) who is the current manager of liverpool fc & jürgen klopp \\
\hline $\mathrm{P} 118_{r}$ (R: league) & 2 & which nrl teams have never won a premiership & new zealand warriors \\
\hline P413 (fielding position) & 2 & what position did ryan tannehill play in college & quarterback \\
\hline P35 (head of state) & 2 & the longest serving samma ruler in sindh was & jam nizamuddin ii \\
\hline P3173 (offers view on) & 2 & where is the leaning tower of pisa in italy located & pisa \\
\hline P7959 (historic county) & 2 & archipelago that includes neolithic settlement of skara brae & orkney \\
\hline P598 $r$ (R: commands) & 2 & union generals civil war army of the potomac & ambrose burnside \\
\hline P306 (operating system) & 2 & what operating system does the macbook pro have & macos \\
\hline P101 (field of work) & 2 & what did robert moog contribute to the music industry in the $1960 \mathrm{~s}$ & electronic music \\
\hline P27 (country of citizenship) & 2 & where is the actress that played wonder woman from & israel \\
\hline P463 (member of) & 2 & what band is the girl from the grinch in & the pretty reckless \\
\hline P4969 (derivative work) & 2 & what is the first book of pretty little liars & pretty little liars \\
\hline P19 (place of birth) & 2 & where did anakin live before he met qui-gon & tatooine \\
\hline P3938 (named by) & 2 & who developed the concept of an iron law of wages & ferdinand lassalle \\
\hline P157 $r$ (R: killed by) & 2 & who does sansa end up with in game of thrones & ramsay bolton \\
\hline P607 (conflict) & 2 & what battle did the tuskegee airmen help win & world war ii \\
\hline $\mathrm{P} 366_{r}$ (R: use) & 2 & what kind of wax are crayons made from & paraffin wax \\
\hline $\mathrm{P}_{551_{r}}(\mathrm{R}:$ residence $)$ & 2 & who lived in the land of nod east of eden & cain \\
\hline P113 (airline hub) & 2 & where does porter airlines fly from in toronto & billy bishop toronto city airport \\
\hline P927 $r$ (R: anatomical location) & 2 & where do the ilium the ischium and the pubis meet & acetabulum \\
\hline P1000 (record held) & 1 & who holds the world record for 100 meters & usain bolt \\
\hline P2541 (operating area) & 1 & what states does the $\mathrm{i}$ pass work in & illinois \\
\hline P4647 (place of first performan) & 1 & where does medea go at the end of the play & athens \\
\hline P483 (studio) & 1 & where was the dark side of the moon recorded & abbey road studios \\
\hline $\mathrm{P} 197_{r}$ (R: adjacent station) & 1 & where does the rocky mountaineer leave from in vancouver & pacific central station \\
\hline P36 $r$ (R: capital) & 1 & what country is in between poland and lithuania & kaliningrad oblast \\
\hline P1589 ${ }_{r}$ (R: lowest point) & 1 & which state is bordered to the north by the artic ocean & alaska \\
\hline P669 (located on street) & 1 & what area of paris is the eiffel tower & champ de mars \\
\hline P1478 (has immediate cause) & 1 & the united states ' war on terror began in the wake of which of the following events & september 11 attacks \\
\hline P1269 ${ }_{r}$ (R: facet of) & 1 & the enlightenment idea of separation of powers included which branches of government & legislature \\
\hline P2679 (author of foreword) & 1 & who wrote the current edition of the catechism & pope john paul ii \\
\hline P669 ${ }_{r}$ (R: located on street) & 1 & where did the beatles take the abbey road picture & abbey road studios \\
\hline P837 $r$ (R: day in year) & 1 & what are three other names for makar sankranti & magh bihu \\
\hline P3113 (does not have part) & 1 & which element in group 1 is not an alkaline metal & hydrogen \\
\hline
\end{tabular}

Table 8: Relation with grounded QA pairs of Natural Questions Training Set (Top 165-246 by frequency). 


\begin{tabular}{|c|c|c|c|}
\hline Relation & Frequency & Question & Answer \\
\hline $\mathrm{P}^{2} 047_{r}$ (R: enemy of) & 1 & who took out the governor's eye on walking dead & michonne \\
\hline $\mathrm{P} 9_{r}$ (R: constellation) & 1 & brightest star in the constellation lyra dan word & vega \\
\hline P3092 (film crew member) & 1 & who pioneered animated movies with his short feature steamboat willie in 1928 & walt disney \\
\hline $\mathrm{P} 2348_{r}(\mathrm{R}:$ time period $)$ & 1 & the main port of axum was the red sea city of & adulis \\
\hline P736 (cover art by) & 1 & who wrote all quite on the western front & erich maria remarque \\
\hline P469 (lakes on river) & 1 & where does the water from the nile come from & lake victoria \\
\hline P205 (basin country) & 1 & in what country would you find the yellow river & china \\
\hline $\mathrm{P} 921_{r}(\mathrm{R}:$ main subject) & 1 & who began the systematic study of political science & american political science review \\
\hline P4934 (calculated from) & 1 & a quantity $15 \mathrm{~m} / \mathrm{s}$ to the north is a measure of & velocity \\
\hline $\mathrm{P} 1411_{r}$ (R: nominated for) & 1 & who won the first oscar for best actress & janet gaynor \\
\hline P4147 (conjugate acid) & 1 & give the name and formula for the acid derived from the following anion chlorite & chlorous acid \\
\hline $\mathrm{P}^{276_{r}}$ (R: location) & 1 & the area between the tigris and euphrates rivers & mesopotamia \\
\hline $\mathrm{P} 413_{r}$ (R: fielding position) & 1 & who has the most clean sheets in the world & iker casillas \\
\hline P710 $r$ (R: participant) & 1 & in the second punic war between carthage and rome carthage formed an alliance with & massylii \\
\hline $\mathrm{P} 2563_{r}$ (R: superpower) & 1 & who taught defence against the dark arts in book number 5 & dolores umbridge \\
\hline P2596 (culture) & 1 & which american civilization was located in a rain forest & maya civilization \\
\hline $\mathrm{P} 1071_{r}$ (R: location of creation) & 1 & where does the young ones develop in humans & uterus \\
\hline $\mathrm{P} 1535_{r}$ (R: used by) & 1 & what programming language is used in microsoft access & visual basic for applications \\
\hline P400 (platform) & 1 & what consoles can you play star wars battlefront on & xbox one \\
\hline P4913 (dialect of) & 1 & what type of arabic is spoken in palestine & south levantine arabic \\
\hline P1066 (student of) & 1 & who is the minister during the regime of chandragupta & chanakya \\
\hline $\mathrm{P} 3342_{r}$ (R: significant person) & 1 & who went before michael jordan in the draft & hakeem olajuwon \\
\hline $\mathrm{P} 6_{r}(\mathrm{R}:$ composer $)$ & 1 & who wrote the power of love celine dion & candy derouge \\
\hline P1427 (start point) & 1 & where did the tour de france start in 1954 & amsterdam \\
\hline P3373 (sibling) & 1 & who is the older brother mario or luigi & mario \\
\hline $\mathrm{P} 2512_{r}$ (R: series spin-off) & 1 & which came first family guy or american dad & family guy \\
\hline $\mathrm{P} 2505_{r}$ (R: carries) & 1 & where does the appalachian trail cross the hudson river & bear mountain bridge \\
\hline P5009 (complies with) & 1 & what type of port is used by flash drives & usb mass storage device class \\
\hline P2094 (competition class) & 1 & what weight class did muhammad ali fight in & heavyweight \\
\hline P1889 $r$ (R: different from) & 1 & what name is given to fats that are liquid at room temperature & oil \\
\hline P7937 (form of creative work) & 1 & wagner's tristan und isolde is an example of & opera \\
\hline P522 (type of orbit) & 1 & what 's the orbit of the international space station & low earth orbit \\
\hline P1303 (instrument) & 1 & what kind of bass does john cooper play & bass guitar \\
\hline P737 $r$ (R: influenced by) & 1 & who are the members of 36 mafia & juicy j \\
\hline P263 (official residence) & 1 & where did zeus spend most of his time & mount olympus \\
\hline P201 (lake outflow) & 1 & where does the water from lake okeechobee drain & caloosahatchee river \\
\hline $\mathrm{P} 178_{r}$ (R: developer) & 1 & operating system developed in 1969 at at\&t 's bell laboratories & unix \\
\hline P1312 (has facet polytope) & 1 & what is the opposite side of a right angle triangle & hypotenuse \\
\hline P20 (place of death) & 1 & where did omri build his new political capital & samaria \\
\hline P2936 (language used) & 1 & what is the national language of saudi arabia & arabic \\
\hline $\mathrm{P} 460{ }_{r}$ (R: said to be the same as) & 1 & what color is a school bus yellow or orange & chrome yellow \\
\hline $\mathrm{P} 682_{r}$ (R: biological process) & 1 & which protein is responsible for the breakdown of a fibrin clot & plasmin \\
\hline P3300 (musical conductor) & 1 & who did the music for ready player one & alan silvestri \\
\hline P547 (commemorates) & 1 & name of ship that landed at plymouth rock & mayflower \\
\hline P2079 (fabrication method) & 1 & the medium of the artwork that decorates the sistine chapel ceiling is & fresco \\
\hline P1037 (director / manager) & 1 & who led the red shirts to victory in sicily & giuseppe garibaldi \\
\hline P972 $2_{r}$ (R: catalog) & 1 & who is number one on america's most wanted & jason derek brown \\
\hline $\mathrm{P} 263_{r}$ (R: official residence) & 1 & which greek god ruled over a gloomy kingdom & hades \\
\hline P2152 (antiparticle) & 1 & a packet or unit of light energy is called a & photon \\
\hline P1462 (standards body) & 1 & who is responsible for creating the standards used on the internet & internet engineering task force \\
\hline $\mathrm{P}_{664_{r}}(\mathrm{R}$ : organizer $)$ & 1 & when did they start using gloves in ufc & ufc 14 \\
\hline P937 (work location) & 1 & where did beethoven live most of his life & vienna \\
\hline P4675 $r$ (R: appears in the form of) & 1 & what was robin 's name in batman and robin & dick grayson \\
\hline $\mathrm{P}^{2596_{r}}$ (R: culture) & 1 & a ruined city on crete centre of the minoan bronze age civilisation & knossos \\
\hline P2554 (production designer) & 1 & who made the movie all dogs go to heaven & don bluth \\
\hline P1038 (relative) & 1 & what is the first name of huey 's dewey 's and louie 's uncle & donald duck \\
\hline P3301 (broadcast by) & 1 & who is broadcasting the super bowl on sunday & $\mathrm{nbc}$ \\
\hline P943 (programmer) & 1 & who wrote the first computer virus called elk cloner & rich skrenta \\
\hline $\mathrm{P} 30_{r}(\mathrm{R}$ : continent) & 1 & is puerto rico in north or central america & puerto rico \\
\hline P135 (movement) & 1 & what kind of art did claude monet paint & impressionism \\
\hline P5051 (towards) & 1 & which part of the cerebral hemisphere is supplied by the middle cerebral artery & cerebrum \\
\hline $\mathrm{P}^{2} 76_{r}$ (R: lyrics by) & 1 & what beatles songs does paul play drums on & dear prudence \\
\hline P364 (original language) & 1 & what language do they speak in kite runner & dari \\
\hline P1071 (location of creation) & 1 & a town in the netherlands known for the production of a tin glazed earthenware & delft \\
\hline $\mathrm{P} 400_{r}$ (R: platform) & 1 & name of the windows phone 8.1 virtual assistant & cortana \\
\hline $\mathrm{P} 452$ (industry) & 1 & what did the hudson bay company do for canada & retail \\
\hline P598 (commands) & 1 & who controlled or ordered the viet cong in combat & hoàng văn thái \\
\hline P1303r $(\mathrm{R}:$ instrument) & 1 & who introduced the bass clarinet as a solo instrument in jazz & herbie mann \\
\hline P3491 (muscle insertion) & 1 & what is the origin and insertion of the semimembranosus & medial condyle of tibia \\
\hline P530 (diplomatic relation) & 1 & which two countries are on the western border of bolivia & chile \\
\hline P1542 (has effect) & 1 & what disease is caused by bacterium treponema pallidum & syphilis \\
\hline P1336 (territory claimed by) & 1 & the falkland islands are off the coast of what south american country & argentina \\
\hline P747 (editions) & 1 & what is the latest ms office for mac & microsoft office 2016 \\
\hline P7153 (significant place) & 1 & on which island is the uss arizona memorial & honolulu \\
\hline P610 $r$ (R: highest point) & 1 & the highest peak in north america $\mathrm{mt}$. mckinley ( or denali ) is located in the state of & alaska \\
\hline P1809 (choreographer) & 1 & who danced the lead role in appalachian spring & martha graham \\
\hline P81 (connecting line) & 1 & what line is parsons green on tube map & district line \\
\hline P122 (type of government) & 1 & what type of government did european settlers create in south africa in 1909 & constitutional monarchy \\
\hline $\mathrm{P}(\mathrm{R}$ : noble title $)$ & 1 & who was crowned the first holy roman emperor & charlemagne \\
\hline $\mathrm{P} 4552$ (mountain range) & 1 & what mountain range is the blue mountains part of & great dividing range \\
\hline P658 (tracklist) & 1 & what was u2 's lead single from ' the joshua tree' & with or without you \\
\hline P195 (collection) & 1 & where is the original star spangled banner located & national museum of american history \\
\hline P609 (terminus location) & 1 & where does route 66 start on the east coast & chicago \\
\hline
\end{tabular}

Table 9: Relation with grounded QA pairs of Natural Questions Training Set (Top 247-329 by frequency). 


\begin{tabular}{|c|c|}
\hline $\begin{array}{l}\text { Triplet } \\
\text { Question } \\
\text { True Passage } \\
\text { Pred. Relation } \\
\text { Pred. Answer }\end{array}$ & $\begin{array}{l}<\text { 'edward heath', ?, "admiral's cup"> } \\
\text { <mask> of edward heath which } 1971 \text { the british prime minister, edward heath, captained one of the winning boats. recent history. } \\
\text {...he captained britain's winning team for the admiral's cup in } 1971 \text { - while prime minister - and also captained the team in the } 1979 \text { fastnet race... } \\
\text { participant in: } 0.33 \text {, winner: } 0.19 \text {, participant: } 0.04 \text {, victory: } 0.03 \text {, sport: } 0.03 \\
\text { admiral's cup }(\checkmark)\end{array}$ \\
\hline $\begin{array}{l}\text { Triplet } \\
\text { Question } \\
\text { True Passage } \\
\text { Pred. Relation } \\
\text { Pred. Answer }\end{array}$ & $\begin{array}{l}<\text { 'scary stories to tell in the dark', 'P110 (illustrated by)', 'stephen gammell'> } \\
<\text { mask> of scary stories to tell in the dark which evocative, nightmarish illustrations for alvin schwartz's "scary stories to tell in the dark" trilogy, he has illustrated nearly seventy } \\
\text { scary stories to tell in the dark is a series of three collections of short horror stories for children, written by alvin schwartz and originally illustrated by stephen gammell... } \\
\text { illustrator: } 0.13 \text {, creator: } 0.11 \text {, author: } 0.07 \text {, editor: } 0.02 \text {, notable work: } 0.02 \\
\text { stephen gammell }(\checkmark)\end{array}$ \\
\hline $\begin{array}{l}\text { Triplet } \\
\text { Question } \\
\text { True Passage } \\
\text { Pred. Relation } \\
\text { Pred. Answer }\end{array}$ & $\begin{array}{l}<\text { 'heeley', ?, 'sheffield tramway'> } \\
<\text { mask> of heeley which first routes, to attercliffe and carbrook, brightside, heeley, nether edge and owlerton opened between } 1873 \\
\text {..sheffield's old tramway stretched from sheffield city centre to woodseats and heeley was at a time the terminus... } \\
\text { located in the administrative territorial entity: } 0.21 \text {, located in the administrative territorial entity: } 0.14 \text {, location: } 0.07 \text {, shares border with: } 0.04 \text {, terminus: } 0.03 \\
\text { old tramway }(\boldsymbol{X})\end{array}$ \\
\hline $\begin{array}{l}\text { Triplet } \\
\text { Question } \\
\text { True Passage } \\
\text { Pred. Relation } \\
\text { Pred. Answer }\end{array}$ & $\begin{array}{l}<\text { 'pablo goncálvez', ?, 'patricia miller (tennis)'> } \\
<\text { mask> of pablo goncálvez which luisa, was the first victim of uruguayan serial killer pablo goncálvez, who suffocated the } 26 \text {-year old to } \\
\text {...the victim was } 26 \text { years old, had a degree in history and a practicing teacher, and was the sister of the well-known tennis player patricia miller... } \\
\text { sibling: } 0.29 \text {, relative: } 0.13 \text {, spouse: } 0.04 \text {, relative: } 0.04 \text {, place of burial: } 0.02 \\
\text { patricia miller }(\checkmark)\end{array}$ \\
\hline $\begin{array}{l}\text { Triplet } \\
\text { Question } \\
\text { True Passage } \\
\text { Pred. Relation } \\
\text { Pred. Answer }\end{array}$ & $\begin{array}{l}<\text { 'chai prakan district', 'P131 }{ }_{r} \text { (R: located in the administrative territorial entity)', 'chai prakan', }> \\
<\text { mask }>\text { of chai prakan district which, is home to the district headquarters of }<\text { mask }><\text { mask }><\text { mask }><\text { mask }>\text { district in the far north of }<\text { mask }>\text { iang m }<\text { mask }>\text { province } \\
\text {..chai prakan is divided into four sub-districts ("tambons"), which are further subdivided into } 44 \text { administrative villages ("muban")... } \\
\text { located in the administrative territorial entity: } 0.43 \text {, capital: } 0.42 \text {, contains administrative territorial entity: } 0.04 \text {, different from: } 0.02 \text {, contains settlement: } 0.01 \\
\text { chai prakarn }(\checkmark)\end{array}$ \\
\hline $\begin{array}{l}\text { Triplet } \\
\text { Question } \\
\text { True Passage } \\
\text { Pred. Relation } \\
\text { Pred. Answer }\end{array}$ & $\begin{array}{l}<\text { 'gothic western', ?, 'lorin morgan-richards'> } \\
<\text { mask> of gothic western which lifestyle and his series "the goodbye family" has been categorized as gothic western. in addition to his work, rich } \\
\text {...in the young adult series, "the goodbye family" by lorin morgan-richards has been considered gothic western with an element of humor... } \\
\text { genre: } 0.92 \text {, movement: } 0.02 \text {, field of work: } 0.0 \text {, genre: } 0.0 \text {, occupation: } 0.0 \\
\text { lorin morgan-richards }(\checkmark)\end{array}$ \\
\hline $\begin{array}{l}\text { Triplet } \\
\text { Question } \\
\text { True Passage } \\
\text { Pred. Relation } \\
\text { Pred. Answer }\end{array}$ & $\begin{array}{l}<\text { 'quentin bell', 'P40 (child)', 'virginia nicholson'> } \\
\text { has kid of quentin bell which her father was the writer and art historian quentin bell, nephew of } \\
\text {...they had three children: julian bell, an artist and muralist; cressida bell, a notable textile designer; and virginia nicholson, the writer of "charleston: a bloomsbury house... } \\
\text { child: } 0.98 \text {, father: } 0.0 \text {, student: } 0.0 \text {, sibling: } 0.0 \text {, relative: } 0.0 \\
\text { virginia nicholson }(\checkmark)\end{array}$ \\
\hline $\begin{array}{l}\text { Triplet } \\
\text { Question } \\
\text { True Passage } \\
\text { Pred. Relation } \\
\text { Pred. Answer }\end{array}$ & $\begin{array}{l}<' \text { take me back to london', 'P361 (part of)', 'no.6 collaborations project'> } \\
<\text { mask> of take me back to london which the border" featuring cabello and cardi b, and "take me back to london" featuring stormzy } \\
\text {...it was released as the eighth single from sheeran's fourth studio album "no.6 collaborations project" (2019)... } \\
\text { part of: } 0.88 \text {, performer: } 0.07 \text {, lyrics by: } 0.01 \text {, producer: } 0.0 \text {, followed by: } 0.0 \\
\text { "no.6 collaborations project }(\checkmark)\end{array}$ \\
\hline $\begin{array}{l}\text { Triplet } \\
\text { Question } \\
\text { True Passage } \\
\text { Pred. Relation } \\
\text { Pred. Answer }\end{array}$ & $\begin{array}{l}<\text { 'u.s. route } 441 \text { in georgia', ?, 'lakemont, georgia'> } \\
<\text { mask }>\text { of u.s. route } 441 \text { in georgia which area between u.s. route } 23 / 441 \text { and }<\text { mask }>\text { rabun. }<\text { mask }><\text { mask }>\text { has a post office with zip code } \\
\text {...from there it passes through the blue ridge mountain communities of wiley, lakemont, and tiger, the latter of which includes... } \\
\text { terminus location: } 0.15 \text {, terminus: } 0.11 \text {, located in the administrative territorial entity: } 0.08 \text {, terminus: } 0.05 \text {, connects with: } 0.03 \\
\text { wiley }(\boldsymbol{X})\end{array}$ \\
\hline $\begin{array}{l}\text { Triplet } \\
\text { Question } \\
\text { True Passage } \\
\text { Pred. Relation } \\
\text { Pred. Answer }\end{array}$ & $\begin{array}{l}<\text { 'anjelica huston', 'P57 } r \text { (R: directed by)', 'agnes browne'> } \\
<\text { mask> of anjelica huston which irish romantic comedy-drama film directed, produced by, and starring anjelica huston, based on the book "the mammy" by brendan o } \\
\text {...her next directorial effort, the irish dramedy "agnes browne" }(\mathbf{1 9 9 9 )} \text {-in which she also starred as the title character- was released to mixed reviews... } \\
\text { terminus location: } 0.15 \text {, terminus: } 0.11 \text {, located in the administrative territorial entity: } 0.08 \text {, terminus: } 0.05 \text {, connects with: } 0.03 \\
\text { "agnes browne }(\checkmark)\end{array}$ \\
\hline $\begin{array}{l}\text { Triplet } \\
\text { Question } \\
\text { True Passage } \\
\text { Pred. Relation } \\
\text { Pred. Answer }\end{array}$ & $\begin{array}{l}<\text { 'cadillac eldorado', ?, 'oldsmobile toronado'> } \\
<\text { mask }>\text { of cadillac eldorado which } 1967 \text {, cadillac adopted its own version of the upp for the cadillac eldor }<\text { mask }>\text {, using the cadillac v8 engine. } \\
\text {...by } 2000 \text {, the eldorado was the last of a dying breed: its buick riviera and oldsmobile toronado stablemates had been discontinued, as had its perennial rival the lincoln mark... } \\
\text { follows: } 0.38 \text {, followed by: } 0.05 \text {, brand: } 0.04 \text {, based on: } 0.02 \text {, subclass of: } 0.02 \\
\text { oldsmobile toronado }(\checkmark)\end{array}$ \\
\hline $\begin{array}{l}\text { Triplet } \\
\text { Question } \\
\text { True Passage } \\
\text { Pred. Relation } \\
\text { Pred. Answer }\end{array}$ & $\begin{array}{l}<\text { 'corsican nuthatch', 'P138 (named after)', 'john whitehead (explorer)'> } \\
\text { eponym of corsican nuthatch which } 82 \text { where he discovered a bird new to science, the corsican nuthatch. white }<\text { mask }>\text { travelled in malacca, north borneo, } \\
\text {...the corsican nuthatch was discovered by the english collector john whitehead in june } 1883 \text { when he shot a specimen while on a trip in the corsican mountains... } \\
\text { named after: } 0.97 \text {, discoverer or inventor: } 0.01 \text {, named after: } 0.0 \text {, different from: } 0.0 \text {, place served by transport hub: } 0.0 \\
\text { john whitehead }(\mathcal{})\end{array}$ \\
\hline $\begin{array}{l}\text { Triplet } \\
\text { Question } \\
\text { True Passage } \\
\text { Pred. Relation } \\
\text { Pred. Answer }\end{array}$ & $\begin{array}{l}<\text { 'mutual information', ?, 'information content'> } \\
<\text { mask> of mutual information which formula_ } 13 \text { is also often used for the related quantity of mutual<mask>, many authors use a lowercase formula_14 for } \\
\text {...it quantifies the "amount of information" (in units such as shannons (bits), nats or hartleys) obtained about one random variable through observing the other random variable... } \\
\text { subclass of: } 0.48 \text {, different from: } 0.08 \text {, opposite of: } 0.06 \text {, subclass of: } 0.05 \text {, said to be the same as: } 0.03 \\
\text { information theory }(\boldsymbol{X})\end{array}$ \\
\hline $\begin{array}{l}\text { Triplet } \\
\text { Question } \\
\text { True Passage } \\
\text { Pred. Relation } \\
\text { Pred. Answer }\end{array}$ & $\begin{array}{l}<\text { 'oculus (film)', 'P272 (production company)', 'intrepid pictures'> } \\
<\text { mask> of oculus (film) which". in may } 2012 \text { filmdistrict acquired the film rights to what would become "oculus". soon after, the film released on april } 11 \\
\text {...eventually, intrepid pictures expressed interest in producing the film "as long as you don't do it found footage".... } \\
\text { production company: } 0.29 \text {, producer: } 0.19 \text {, distributed by: } 0.07 \text {, screenwriter: } 0.04 \text {, director: } 0.03 \\
\text { intrepid pictures }(\checkmark)\end{array}$ \\
\hline
\end{tabular}

Table 10: Examples of generated Relational QA datapoints and the predicted relation and answer by DPR pre-trained via RGPT-QA. 


\begin{tabular}{|c|c|c|c|c|c|}
\hline Relation Name & Freq & Question & True Answer & RGPT-QA Prediction & Supervised DPR Prediction \\
\hline $\begin{array}{l}\text { R: based on } \\
\text { subject has role } \\
\text { practiced by } \\
\text { industry } \\
\text { made from } \\
\text { offers view on } \\
\text { R: residence } \\
\text { mother } \\
\text { R: employer } \\
\text { R: has part } \\
\text { indigenous to } \\
\text { river source } \\
\text { tributary } \\
\text { R: family } \\
\text { R: genre } \\
\text { R: genre } \\
\text { parent organization } \\
\text { narrator } \\
\text { educated at } \\
\text { director } \\
\text { executive producer } \\
\text { R: player of } \\
\text { R: player of } \\
\text { shares border with } \\
\text { depicts } \\
\text { depicts } \\
\text { R: notable work } \\
\text { presenter } \\
\text { presenter } \\
\text { material used } \\
\text { main subject } \\
\text { instance of } \\
\text { instance of } \\
\text { country of origin } \\
\text { R: subclass of } \\
\text { R: subclass of } \\
\text { located in } \\
\text { R: part of } \\
\text { R: part of } \\
\text { characters } \\
\text { R: award received } \\
\text { participating team } \\
\text { spouse } \\
\text { R: instance of } \\
\text { R: instance of } \\
\text { R: instance of } \\
\text { named after } \\
\text { part of } \\
\text { part of } \\
\text { officeholder } \\
\text { participant } \\
\text { participant } \\
\text { participant } \\
\text { filming location } \\
\text { filming location }\end{array}$ & $\begin{array}{l}0 \\
0 \\
0 \\
1 \\
2 \\
2 \\
2 \\
2 \\
3 \\
3 \\
3 \\
4 \\
5 \\
5 \\
6 \\
6 \\
8 \\
10 \\
10 \\
15 \\
16 \\
16 \\
16 \\
16 \\
19 \\
19 \\
19 \\
21 \\
21 \\
22 \\
24 \\
29 \\
29 \\
37 \\
44 \\
44 \\
46 \\
49 \\
49 \\
50 \\
51 \\
52 \\
53 \\
56 \\
56 \\
56 \\
64 \\
70 \\
70 \\
87 \\
88 \\
88 \\
88 \\
98 \\
98\end{array}$ & 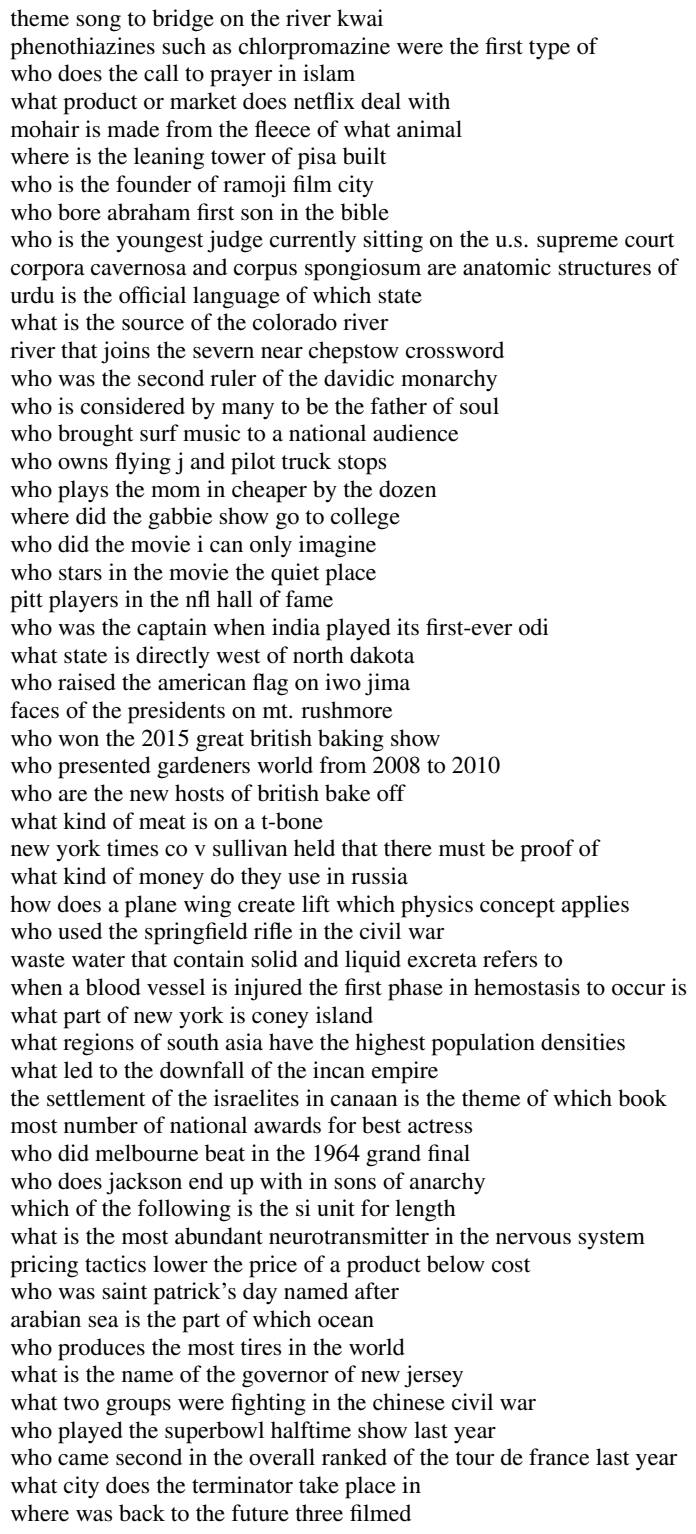 & $\begin{array}{l}\text { the river kwai march } \\
\text { antipsychotic } \\
\text { muezzin } \\
\text { streaming media } \\
\text { angora goat } \\
\text { pisa } \\
\text { ramoji rao } \\
\text { hagar } \\
\text { neil gorsuch } \\
\text { penis } \\
\text { pakistan } \\
\text { la poudre pass } \\
\text { river wye } \\
\text { solomon } \\
\text { james brown } \\
\text { the beach boys } \\
\text { pilot corporation } \\
\text { bonnie hunt } \\
\text { university of pittsburgh } \\
\text { erwin brothers } \\
\text { john krasinski } \\
\text { mike ditka } \\
\text { ajit wadekar } \\
\text { montana } \\
\text { michael strank } \\
\text { abraham lincoln } \\
\text { nadiya hussain } \\
\text { toby buckland } \\
\text { noel fielding } \\
\text { beef } \\
\text { actual malice } \\
\text { kopeks } \\
\text { force } \\
\text { united states } \\
\text { sewage } \\
\text { coagulation } \\
\text { brooklyn } \\
\text { philippines } \\
\text { battle of cajamarca } \\
\text { joshua } \\
\text { shabana azmi } \\
\text { collingwood football club } \\
\text { tara knowles } \\
\text { metre } \\
\text { serotonin } \\
\text { loss leader } \\
\text { saint patrick } \\
\text { indian ocean } \\
\text { lego } \\
\text { phil murphy } \\
\text { communist party of china } \\
\text { bruno mars } \\
\text { rigoberto urán } \\
\text { los angeles } \\
\text { monument valley } \\
\text { and }\end{array}$ & $\begin{array}{l}\text { the river kwai march } \\
\text { psychiatry } \\
\text { muezzin } \\
\text { streaming media } \\
\text { angora goat } \\
\text { pisa } \\
\text { ramoji rao } \\
\text { sarah } \\
\text { neil gorsuch } \\
\text { penis } \\
\text { jharkhand } \\
\text { la poudre pass } \\
\text { river lugg } \\
\text { jeroboam } \\
\text { james brown } \\
\text { the beach boys } \\
\text { pilot corporation } \\
\text { bonnie hunt } \\
\text { university of pittsburgh } \\
\text { the erwin brothers } \\
\text { john krasinski } \\
\text { ruben brown } \\
\text { srinivasaraghavan } \\
\text { montana } \\
\text { ira hayes } \\
\text { thomas jefferson } \\
\text { joanne wheatley } \\
\text { joe swift } \\
\text { noel fielding } \\
\text { cut from the short loin } \\
\text { malice } \\
\text { ruble or rouble } \\
\text { newton's second law } \\
\text { marine corps } \\
\text { sewage } \\
\text { wound healing } \\
\text { brooklyn } \\
\text { philippines } \\
\text { captured } \\
\text { the book of joshua } \\
\text { five } \\
\text { collingwood } \\
\text { tara knowles } \\
\text { meter } \\
\text { serotonin } \\
\text { loss leader } \\
\text { saint patrick } \\
\text { northern indian ocean } \\
\text { lego tire: lego tire a lego } \\
\text { phil murphy } \\
\text { communist party of china } \\
\text { beyoncé } \\
\text { rigoberto urán } \\
\text { los angeles } \\
\text { monument valley } \\
\text { and }\end{array}$ & $\begin{array}{l}\text { march } \\
\text { medication } \\
\text { mosque } \\
\text { netflix } \\
\text { goat } \\
\text { pisa the leaning tower of pisa } \\
\text { telugu film producer ramoji rao } \\
\text { yishma'el } \\
\text { leonard i. garth } \\
\text { corpus cavernosum } \\
\text { jammu and kashmir } \\
\text { colorado begins at la poudre pass } \\
\text { lugg } \\
\text { solomon's son, rehoboam } \\
\text { sam cooke } \\
\text { dean } \\
\text { berkshire hathaway } \\
\text { kate } \\
\text { the university of pittsburgh } \\
\text { bart millard } \\
\text { emily blunt } \\
\text { tony dorsett } \\
\text { s } \\
\text { manitoba } \\
\text { rene gagnon, ira hayes } \\
\text { theodore roosevelt } \\
\text { edd kimber } \\
\text { carol klein and joe swift } \\
\text { sandi toksvig } \\
\text { tenderloin } \\
\text { truth } \\
\text { the russian ruble or rouble } \\
\text { reaction force } \\
\text { army } \\
\text { pathogens } \\
\text { endothelial injury } \\
\text { borough of brooklyn } \\
\text { indonesia } \\
\text { victory } \\
\text { book of joshua } \\
\text { three } \\
\text { melbourne football club } \\
\text { opie winston } \\
\text { litre } \\
\text { glutamate } \\
\text { increase in profits } \\
\text { saint patrick } \\
\text { the northern indian ocean } \\
\text { lego blocks. lego } \\
\text { democrat phil murphy } \\
\text { republic of china } \\
\text { coldplay } \\
\text { chris froome } \\
\text { hemdale } \\
\text { jamestown, california }\end{array}$ \\
\hline
\end{tabular}

Table 11: Comparison of the prediction of DPR initialized by RGPT-QA with DPR without pre-training. These are all samples that two models made different predictions, and the relation frequency in the training set is less than 100. 


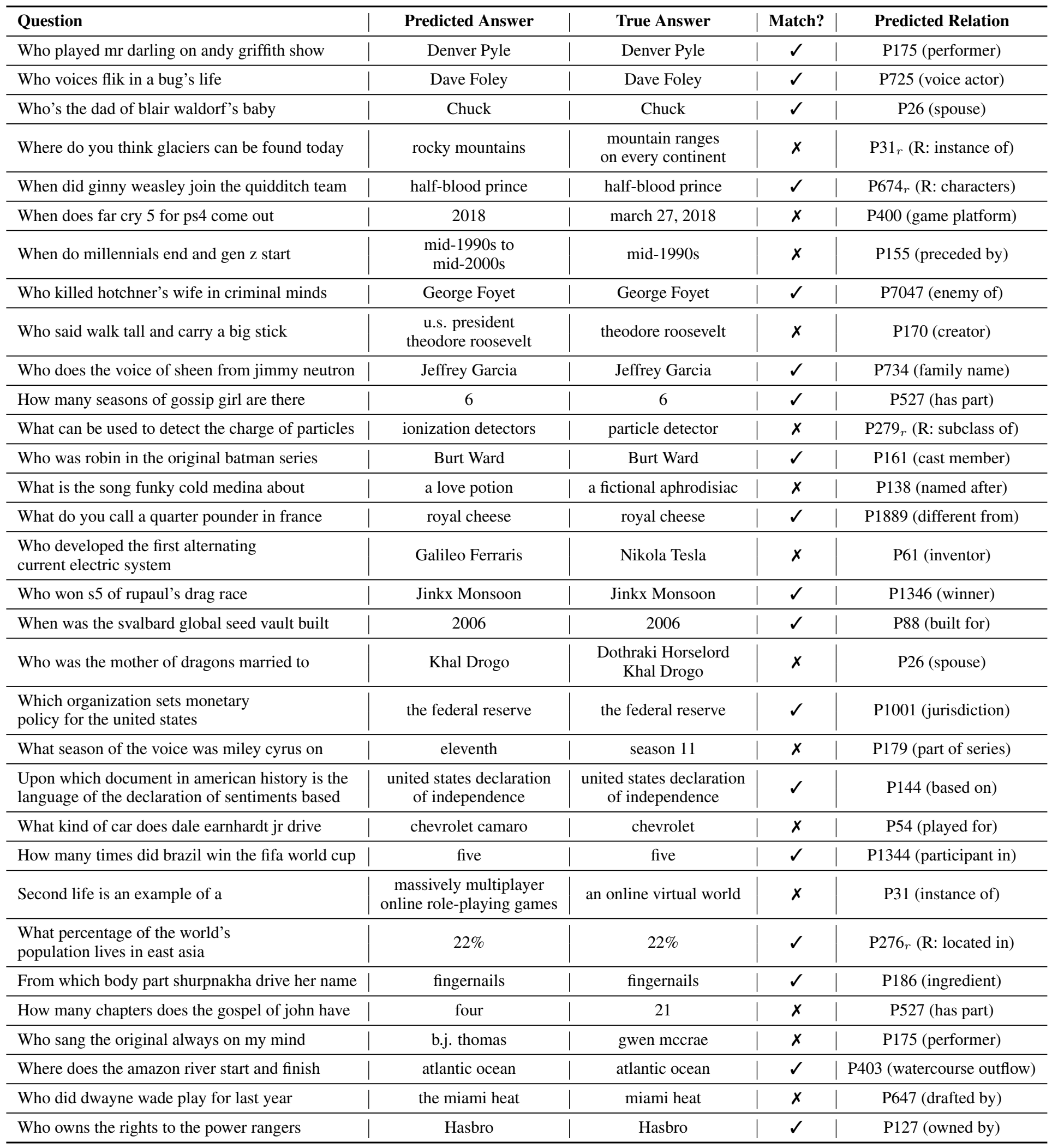

Table 12: Predicted relations for those QA pairs in Natural Questions Valid Set that cannot be aligned to WikiData. 